\title{
General representations for wavefield modeling and inversion in geophysics
}

\author{
Kees Wapenaar ${ }^{1}$
}

\begin{abstract}
Acoustic, electromagnetic, elastodynamic, poroelastic, and electroseismic waves are all governed by a unified matrix-vector wave equation. The matrices in this equation obey the same symmetry properties for each of these wave phenomena. This implies that the wave vectors for each of these phenomena obey the same reciprocity theorems. By substituting Green's matrices in these reciprocity theorems, unified wavefield representations are obtained. Analogous to the well-known acoustic wavefield representations, these unified representations find applications in geophysical modeling, migration, inversion, multiple elimination, and interferometry.
\end{abstract}

\section{INTRODUCTION}

Wavefield representations play an important role in forward and inverse geophysical problems, such as modeling, migration, inversion, multiple elimination, and, recently, interferometry. Various authors have derived acoustic and elastodynamic wavefield representations by substituting Green's functions into the Rayleigh and Rayleigh-Betti reciprocity theorems, respectively (Morse and Feshbach, 1953; de Hoop, 1958; Gangi, 1970, 2000; Aki and Richards, 1980; Fokkema and van den Berg, 1993). In this paper, we follow a similar approach for a general matrix-vector wave equation that governs acoustic, electromagnetic, elastodynamic, poroelastic, or electroseismic wave propagation. First, we derive general convolution and correlation reciprocity theorems for this wave equation, supplemented with boundary conditions for imperfectly coupled interfaces. Next, we introduce a Green's matrix as the point-source solution of the general wave equation. By substituting this Green's matrix into the reciprocity theorems, we obtain general convolution- and correlation-type representations. We conclude this paper by briefly discussing a number of applications of these general representations in seismic modeling, migration, inversion, multiple elimination, and interferometry.

\section{MATRIX-VECTOR WAVE EQUATION}

Diffusion, flow, and wave phenomena can each be captured by the differential matrix-vector equation,

$$
\mathbf{A} D_{t} \mathbf{u}+\mathbf{B u}+\mathbf{D}_{\mathbf{x}} \mathbf{u}=\mathbf{s}
$$

(Wapenaar and Fokkema, 2004), where $\mathbf{u}=\mathbf{u}(\mathbf{x}, t)$ is a vector containing space- and time-dependent field quantities, $\mathbf{s}=\mathbf{s}(\mathbf{x}, t)$ is a source vector, $\mathbf{A}=\mathbf{A}(\mathbf{x})$ and $\mathbf{B}=\mathbf{B}(\mathbf{x})$ are matrices containing space-dependent material parameters, and $\mathbf{D}_{\mathbf{x}}$ is a matrix containing the spatial differential operators $\partial_{1}, \partial_{2}$, and $\partial_{3}$. $D_{t}$ denotes the material time derivative, defined as $D_{t}=\partial_{t}+\mathbf{v}^{0} \cdot \boldsymbol{\nabla}=\partial_{t}+v_{k}^{0} \partial_{k}$, where $\partial_{t}$ is the time derivative in the reference frame and $\mathbf{v}^{0}=\mathbf{v}^{0}(\mathbf{x})$ the spacedependent flow velocity of the material; $v_{k}^{0}$ denotes the $k$ th component of $\mathbf{v}^{0}$. Einstein's summation convention applies to repeated subscripts; lower-case Latin subscripts (except $t$ ) run from 1 to 3. In exploration geophysics, we consider nonmoving media; hence, from here, onward we replace $D_{t}$ by $\partial_{t}$.

For acoustic wave propagation in an attenuating fluid, the vectors and matrices in equation 1 are defined by

$$
\mathbf{u}=\left(\begin{array}{c}
p \\
v_{1} \\
v_{2} \\
v_{3}
\end{array}\right), \quad \mathbf{s}=\left(\begin{array}{c}
q \\
f_{1} \\
f_{2} \\
f_{3}
\end{array}\right),
$$

with $p=p(\mathbf{x}, t)$ the acoustic pressure, $v_{i}=v_{i}(\mathbf{x}, t)$ the particle velocity, $q=q(\mathbf{x}, t)$ the volume injection rate, $f_{i}=f_{i}(\mathbf{x}, t)$ the external force;

Manuscript received by the Editor December 16, 2006; revised manuscript received March 28, 2007; published online August $23,2007$.

${ }^{1}$ Delft University of Technology, Department of Geotechnology, Delft, the Netherlands. Email: c.p.a.wapenaar@tudelft.nl (C) 2007 Society of Exploration Geophysicists. All rights reserved. 


$$
\mathbf{A}=\left(\begin{array}{cccc}
\kappa & 0 & 0 & 0 \\
0 & \rho & 0 & 0 \\
0 & 0 & \rho & 0 \\
0 & 0 & 0 & \rho
\end{array}\right), \quad \mathbf{B}=\left(\begin{array}{cccc}
b^{p} & 0 & 0 & 0 \\
0 & b^{v} & 0 & 0 \\
0 & 0 & b^{v} & 0 \\
0 & 0 & 0 & b^{v}
\end{array}\right),
$$

with $\kappa=\kappa(\mathbf{x})$ the compressibility, $\rho=\rho(\mathbf{x})$ the mass density, $b^{p}$ $=b^{p}(\mathbf{x})$ and $b^{v}=b^{v}(\mathbf{x})$ the loss terms, and

$$
\mathbf{D}_{\mathbf{x}}=\left(\begin{array}{cccc}
0 & \partial_{1} & \partial_{2} & \partial_{3} \\
\partial_{1} & 0 & 0 & 0 \\
\partial_{2} & 0 & 0 & 0 \\
\partial_{3} & 0 & 0 & 0
\end{array}\right)
$$

For electromagnetic diffusion and/or wave propagation in matter, we have

$$
\mathbf{u}=\left(\begin{array}{l}
\mathbf{E} \\
\mathbf{H}
\end{array}\right), \quad \mathbf{s}=\left(\begin{array}{c}
-\mathbf{J}^{e} \\
-\mathbf{J}^{m}
\end{array}\right)
$$

with $\mathbf{E}=\mathbf{E}(\mathbf{x}, t)$ and $\mathbf{H}=\mathbf{H}(\mathbf{x}, t)$ the electric and magnetic field vectors, $\mathbf{J}^{e}=\mathbf{J}^{e}(\mathbf{x}, t)$ and $\mathbf{J}^{m}=\mathbf{J}^{m}(\mathbf{x}, t)$ the external electric and magnetic current density vectors;

$$
\mathbf{A}=\left(\begin{array}{cc}
\boldsymbol{\epsilon} & \mathbf{O} \\
\mathbf{O} & \boldsymbol{\mu}
\end{array}\right), \quad \mathbf{B}=\left(\begin{array}{cc}
\boldsymbol{\sigma}^{e} & \mathbf{O} \\
\mathbf{O} & \boldsymbol{\sigma}^{m}
\end{array}\right),
$$

with $\boldsymbol{\epsilon}=\boldsymbol{\epsilon}(\mathbf{x})$ and $\boldsymbol{\mu}=\boldsymbol{\mu}(\mathbf{x})$ the permittivity and permeability tensors, $\boldsymbol{\sigma}^{e}=\boldsymbol{\sigma}^{e}(\mathbf{x})$ and $\boldsymbol{\sigma}^{m}=\boldsymbol{\sigma}^{m}(\mathbf{x})$ the electric and magnetic conductivity tensors, $\mathbf{O}$ the null-matrix, and

$$
\mathbf{D}_{\mathbf{x}}=\left(\begin{array}{cc}
\mathbf{O} & \mathbf{D}_{0}^{T} \\
\mathbf{D}_{0} & \mathbf{O}
\end{array}\right), \quad \mathbf{D}_{0}=\left(\begin{array}{ccc}
0 & -\partial_{3} & \partial_{2} \\
\partial_{3} & 0 & -\partial_{1} \\
-\partial_{2} & \partial_{1} & 0
\end{array}\right)
$$

(superscript $T$ denotes matrix transposition only; it does not denote operator transposition).

For elastodynamic wave propagation in a solid, we have $\mathbf{u}^{T}$ $=\left(\mathbf{v}^{T},-\boldsymbol{\tau}_{1}^{T},-\boldsymbol{\tau}_{2}^{T},-\boldsymbol{\tau}_{3}^{T}\right)$ (with $\mathbf{v}$ and $\boldsymbol{\tau}_{i}$ the particle velocity and traction vectors), $\mathbf{s}^{T}=\left(\mathbf{f}^{T}, \mathbf{h}_{1}^{T}, \mathbf{h}_{2}^{T}, \mathbf{h}_{3}^{T}\right)$ (with $\mathbf{f}$ and $\mathbf{h}_{i}$ the external force and deformation rate vectors), and $\mathbf{A}, \mathbf{B}$, and $\mathbf{D}_{\mathbf{x}}$ are $12 \times 12$ matrices (de Hoop and de Hoop, 2000; Wapenaar and Fokkema, 2004).

For electroseismic wave propagation in a saturated porous solid (Pride, 1994), we have $\mathbf{u}^{T}=\left(\mathbf{E}^{T}, \mathbf{H}^{T},\left\{\mathbf{v}^{s}\right\}^{T},-\boldsymbol{\tau}_{1}^{T},-\boldsymbol{\tau}_{2}^{T},-\boldsymbol{\tau}_{3}^{T}, \mathbf{w}^{T}, p^{f}\right)$ (with superscripts $s$ and $f$ referring to the solid and fluid phase, respectively), where $\mathbf{E}$ and $\mathbf{H}$ are the average electric and magnetic field vectors, $\mathbf{v}^{s}$ and $\boldsymbol{\tau}_{i}$ the solid particle velocity and bulk traction vectors, $\mathbf{w}=\varphi\left(\mathbf{v}^{f}-\mathbf{v}^{s}\right)$ the filtration velocity (with $\varphi$ the porosity), and $p^{f}$ the fluid pressure. Moreover, $\mathbf{s}^{T}=\left(-\left\{\mathbf{J}^{e}\right\}^{T}\right.$, - $\left.\left\{\mathbf{J}^{m}\right\}^{T}, \mathbf{f}^{T}, \mathbf{h}_{1}^{T}, \mathbf{h}_{2}^{T}, \mathbf{h}_{3}^{T},\left\{\mathbf{f}^{f}\right\}^{T}, h^{f}\right)$, where $\mathbf{J}^{e}$ and $\mathbf{J}^{m}$ are again the external electric and magnetic current density vectors, $\mathbf{f}$ and $\mathbf{f}^{f}$ the external forces on the bulk and on the fluid, and $\mathbf{h}_{i}$ and $h^{f}$ the modified external deformation rates for the bulk and the fluid. Finally, for this situation $\mathbf{A}, \mathbf{B}$, and $\mathbf{D}_{\mathbf{x}}$ are $22 \times 22$ matrices. Omitting $\mathbf{E}, \mathbf{H}, \mathbf{J}^{e}$, and $\mathbf{J}^{m}$ from $\mathbf{u}$ and $\mathbf{s}$ gives the field and source vectors for poroelastic waves (Biot, 1956) governed by $16 \times 16$ matrices $\mathbf{A}, \mathbf{B}$, and $\mathbf{D}_{\mathbf{x}}$. On the other hand, omitting $\mathbf{w}, p^{f}, \mathbf{f}^{f}$ and $h^{f}$ and reorganizing $\mathbf{B}$ results in the electrokinetic equations for a piezoelectric system (Auld, 1973), with $18 \times 18$ matrices $\mathbf{A}, \mathbf{B}$ and $\mathbf{D}_{\mathbf{x}}$.
In all cases, matrices $\mathbf{A}(\mathbf{x})$ and $\mathbf{B}(\mathbf{x})$ can be replaced by convolutional operators $\mathbf{A}(\mathbf{x}, t) *$ and $\mathbf{B}(\mathbf{x}, t) *$ to account for more general attenuation mechanisms.

We define the temporal Fourier transform of a space- and time-dependent quantity $p(\mathbf{x}, t)$ as

$$
\hat{p}(\mathbf{x}, \omega)=\int_{-\infty}^{\infty} \exp (-j \omega t) p(\mathbf{x}, t) \mathrm{d} t,
$$

where $j$ is the imaginary unit and $\omega$ the angular frequency. Applying the Fourier transform to all terms in matrix-vector equation 1, with $D_{t}$ replaced by $\partial_{t}$ and $\mathbf{A}(\mathbf{x})$ and $\mathbf{B}(\mathbf{x})$ replaced by convolutional operators $\mathbf{A}(\mathbf{x}, t) *$ and $\mathbf{B}(\mathbf{x}, t) *$, we obtain

$$
j \omega \hat{\mathbf{A}} \hat{\mathbf{u}}+\hat{\mathbf{B}} \hat{\mathbf{u}}+\mathbf{D}_{\mathbf{x}} \hat{\mathbf{u}}=\hat{\mathbf{s}},
$$

where, apart from the field and source vectors $\hat{\mathbf{u}}(\mathbf{x}, \omega)$ and $\hat{\mathbf{s}}(\mathbf{x}, \omega)$, the material parameter matrices $\hat{\mathbf{A}}(\mathbf{x}, \omega)$ and $\hat{\mathbf{B}}(\mathbf{x}, \omega)$ are in their general form frequency-dependent and complex-valued. Note that $j \omega \hat{\mathbf{A}}$ and $\hat{\mathbf{B}}$ could be combined into one material-parameter matrix. However, we prefer to keep these terms separated to acknowledge the different character of the parameters in $\hat{\mathbf{A}}$ and $\hat{\mathbf{B}}$.

For each situation, matrices $\hat{\mathbf{A}}, \hat{\mathbf{B}}$, and $\mathbf{D}_{\mathbf{x}}$ obey the symmetry relations

$$
\begin{aligned}
\mathbf{K} \hat{\mathbf{A}} \mathbf{K} & =\hat{\mathbf{A}}=\hat{\mathbf{A}}^{T}, \\
\mathbf{K} \hat{\mathbf{B}} \mathbf{K} & =\hat{\mathbf{B}}^{T}, \\
\mathbf{K D}_{\mathbf{x}} \mathbf{K} & =-\mathbf{D}_{\mathbf{x}}=-\mathbf{D}_{\mathbf{x}}^{T},
\end{aligned}
$$

where $\mathbf{K}$ is a real-valued diagonal matrix, obeying the property $\mathbf{K}$ $=\mathbf{K}^{-1}$. For example, for the acoustic and electromagnetic situations discussed above, we have

$\mathbf{K}=\left(\begin{array}{cccc}1 & 0 & 0 & 0 \\ 0 & -1 & 0 & 0 \\ 0 & 0 & -1 & 0 \\ 0 & 0 & 0 & -1\end{array}\right) \quad$ and $\mathbf{K}=\left(\begin{array}{cc}-\mathbf{I} & \mathbf{O} \\ \mathbf{O} & \mathbf{I}\end{array}\right)$,

respectively, with $\mathbf{I}$ the identity matrix.

\section{MATRIX-VECTOR BOUNDARY CONDITION}

At any position in space where the medium parameters in matrices $\hat{\mathbf{A}}$ and $\hat{\mathbf{B}}$ are discontinuous, the wavefield quantities in vector $\hat{\mathbf{u}}$ should obey boundary conditions. The same is true at a fracture with imperfect coupling. For this situation, the wavefield quantities in $\hat{\mathbf{u}}$ may exhibit a finite jump. We call both types of medium singularities interfaces. In the following, we consider the most general case for which the medium parameters are different at both sides of the interface and the media are not in perfect contact with each other.

Consider an interface with normal vector $\mathbf{n}=\left(n_{1}, n_{2}, n_{3}\right)^{T}$ between two materials (see Figure 1). In linearized form, the boundary conditions at an imperfect interface can be formulated in the space-frequency domain as

$$
[\mathbf{M} \hat{\mathbf{u}}]=-j \omega \hat{\mathbf{Y}}\langle\mathbf{M} \hat{\mathbf{u}}\rangle
$$

(Wapenaar et al., 2004), where $\mathbf{M}$ is a matrix that contracts the wave vector $\hat{\mathbf{u}}$ to the components that are involved in the boundary condi- 
tions, $\hat{\mathbf{Y}}=\hat{\mathbf{Y}}(\mathbf{x}, \omega)$ is a matrix containing the boundary parameters, and $[\cdot]$ and $\langle\cdot\rangle$ represent the jump and the average across the interface, respectively, as stated by

$$
\begin{gathered}
{[\hat{p}(\mathbf{x}, \omega)]=\lim _{h \downarrow 0}(\hat{p}(\mathbf{x}+h \mathbf{n}, \omega)-\hat{p}(\mathbf{x}-h \mathbf{n}, \omega)),} \\
\langle\hat{p}(\mathbf{x}, \omega)\rangle=\lim _{h \downarrow 0}(\hat{p}(\mathbf{x}+h \mathbf{n}, \omega)+\hat{p}(\mathbf{x}-h \mathbf{n}, \omega)) / 2,
\end{gathered}
$$

where $\mathbf{x}$ is chosen at the interface.

For acoustic waves, the matrices in equation 14 are defined as

$$
\mathbf{M}=\left(\begin{array}{cccc}
1 & 0 & 0 & 0 \\
0 & n_{1} & n_{2} & n_{3}
\end{array}\right) \quad \text { and } \hat{\mathbf{Y}}=\left(\begin{array}{cc}
0 & \hat{\rho}^{\mathrm{b}} \\
\hat{\kappa}^{\mathrm{b}} & 0
\end{array}\right) .
$$

The superscript $\mathrm{b}$ denotes that $\hat{\rho}^{\mathrm{b}}=\hat{\rho}^{\mathrm{b}}(\mathbf{x}, \omega)$ and $\hat{\kappa}^{\mathrm{b}}=\hat{\kappa}^{\mathrm{b}}(\mathbf{x}, \omega)$ are boundary parameters. The dimension of each boundary parameter is equal to the dimension of the corresponding volumetric parameter, multiplied by meter. For vanishing $\hat{\rho}^{\mathrm{b}}$ and $\hat{\kappa}^{\mathrm{b}}$, equation 14 , with $\hat{\mathbf{u}}, \mathbf{M}$, and $\hat{\mathbf{Y}}$ defined in equations 2 and 17, reduces to the standard boundary conditions for perfectly coupled fluids, i.e., $[\hat{p}]=0$ and $\left[\hat{v}_{i} n_{i}\right]$ $=0$. When $\hat{\rho}^{\mathrm{b}}$ and $\hat{\kappa}^{\mathrm{b}}$ are nonzero, $1 / j \omega \hat{\rho}^{\mathrm{b}}$ is the hydraulic boundary permeability, $\hat{\kappa}^{\mathrm{b}}$ the boundary compressibility, and $1 / \hat{\kappa}^{\mathrm{b}}=\hat{K}^{\mathrm{b}}$ the boundary stiffness. Note that the dimension of the boundary stiffness $\hat{K}^{\mathrm{b}}$ is that of stiffness per meter (i.e., $\mathrm{Pa} / \mathrm{m}$ ). Therefore, $\hat{K}^{\mathrm{b}}$ is also called the specific boundary stiffness.

For elastodynamic waves, the matrices in equation 14 are defined as

$$
\mathbf{M}=\left(\begin{array}{cccc}
\mathbf{I} & \mathbf{O} & \mathbf{O} & \mathbf{O} \\
\mathbf{O} & n_{1} \mathbf{I} & n_{2} \mathbf{I} & n_{3} \mathbf{I}
\end{array}\right) \quad \text { and } \hat{\mathbf{Y}}=\left(\begin{array}{cc}
\mathbf{O} & \hat{\mathbf{S}}^{\mathrm{b}} \\
\hat{\boldsymbol{\rho}}^{\mathrm{b}} & \mathbf{O}
\end{array}\right),
$$

where $\hat{\boldsymbol{\rho}}^{\mathrm{b}}$ and $\hat{\mathbf{S}}^{\mathrm{b}}$ are the boundary density and compliance tensors, respectively. When $\hat{\boldsymbol{\rho}}^{\mathrm{b}}=\mathbf{O}$ (which is usually a good approximation) and $\mathbf{n}=(0,0,1)^{T}$ (i.e., the interface is horizontal), equation 14 , with $\mathbf{M}$ and $\hat{\mathbf{Y}}$ defined in equation 18, reduces to the linear slip model of Schoenberg (1980) when $\hat{\mathbf{S}}^{\mathrm{b}}$ is diagonal and real-valued, to the extended linear slip model of Pyrak-Nolte et al. (1990) when $\hat{\mathbf{S}}^{\mathrm{b}}$ is diagonal and complex-valued, or to the general boundary model (including shear-induced conversion) of Nakagawa et al. (2000) when the nondiagonal elements of $\hat{\mathbf{S}}^{\mathrm{b}}$ are also nonzero. Liu et al. $(1995,2000)$ relate the parameters in the compliance tensor to the details of the microstructure of the interface.

For electromagnetic waves, equation 14 is a generalization of the Kaufman and Keller (1983) conductive interface model. For poroelastic waves, it is a generalization of the Gurevich and Schoenberg (1999) permeable interface model; when $\hat{\mathbf{Y}}$ vanishes, it reduces to the Deresiewicz and Skalak (1963) open-pore boundary condition

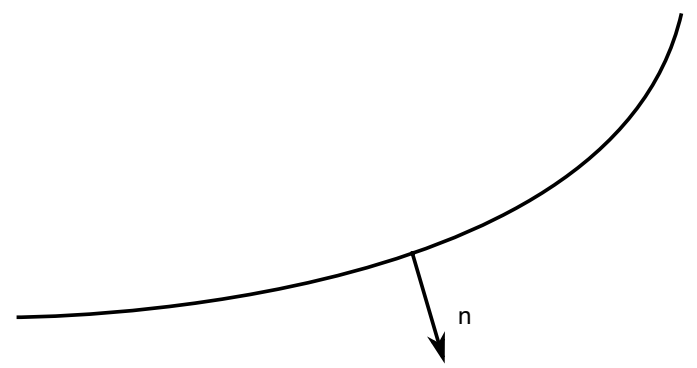

Figure 1. Interface between two media with imperfect coupling. for the perfectly coupled porous solids. For electroseismic waves, equation 14 combines the boundary conditions for electromagnetic and poroelastic waves.

In all cases $\hat{\mathbf{Y}}$ obeys the symmetry relations

$$
\hat{\mathbf{Y}}^{T} \mathbf{N}=-\mathbf{N} \hat{\mathbf{Y}} \quad \text { and } \hat{\mathbf{Y}}^{\dagger} \mathbf{J}=\mathbf{J} \hat{\mathbf{Y}}^{*}
$$

(Wapenaar et al., 2004), where superscript $*$ denotes complex conjugation and $\dagger$ complex conjugation and transposition. For example, for the acoustic and elastodynamic situations discussed above, we have

$$
\mathbf{N}=\left(\begin{array}{cc}
0 & 1 \\
-1 & 0
\end{array}\right), \quad \mathbf{J}=\left(\begin{array}{ll}
0 & 1 \\
1 & 0
\end{array}\right)
$$

and

$$
\mathbf{N}=\left(\begin{array}{cc}
\mathbf{O} & \mathbf{I} \\
-\mathbf{I} & \mathbf{O}
\end{array}\right), \quad \mathbf{J}=\left(\begin{array}{cc}
\mathbf{O} & \mathbf{I} \\
\mathbf{I} & \mathbf{O}
\end{array}\right)
$$

respectively.

\section{CONVOLUTION-TYPE RECIPROCITY THEOREM}

In general, a reciprocity theorem interrelates two independent states in one and the same domain (de Hoop, 1966; Fokkema and van de Berg, 1993). Here, we derive a reciprocity theorem for the general wave vector $\hat{\mathbf{u}}$ described in the previous sections. We introduce two independent states (i.e., wavefields, medium parameters, boundary parameters, and source functions) that will be distinguished by the subscripts $A$ and $B$, (see Table 1). In the frequency domain, each of these states obeys the general matrix-vector-wave equation 9 . We consider the interaction quantity $\hat{\mathbf{u}}_{A}^{T} \mathbf{K} \mathbf{D}_{\mathbf{x}} \hat{\mathbf{u}}_{B}-\left(\mathbf{D}_{\mathbf{x}} \hat{\mathbf{u}}_{A}\right)^{T} \mathbf{K} \hat{\mathbf{u}}_{B}$. Using wave equation 9 as well as symmetry relations 10 and 11 for both states, we obtain

$$
\begin{aligned}
\hat{\mathbf{u}}_{A}^{T} \mathbf{K} \mathbf{D}_{\mathbf{x}} \hat{\mathbf{u}}_{B}-\left(\mathbf{D}_{\mathbf{x}} \hat{\mathbf{u}}_{A}\right)^{T} \mathbf{K} \hat{\mathbf{u}}_{B}= & \hat{\mathbf{u}}_{A}^{T} \mathbf{K} \hat{\mathbf{s}}_{B}-\hat{\mathbf{s}}_{A}^{T} \mathbf{K} \hat{\mathbf{u}}_{B} \\
& -\hat{\mathbf{u}}_{A}^{T} \mathbf{K}\left\{j \omega\left(\hat{\mathbf{A}}_{B}-\hat{\mathbf{A}}_{A}\right)\right. \\
& \left.+\left(\hat{\mathbf{B}}_{B}-\hat{\mathbf{B}}_{A}\right)\right\} \hat{\mathbf{u}}_{B} .
\end{aligned}
$$

This is the local form of the convolution-type matrix-vector reciprocity theorem. We call this convolution type because the products in the frequency domain $\left(\hat{\mathbf{u}}_{A}^{T} \mathbf{K} \hat{\mathbf{s}}_{B}\right.$ etc.) correspond to convolutions in the time domain. Next, we consider an arbitrary spatial domain $\mathbb{D}$ with boundary $\partial \mathrm{D}$ and outward-pointing normal vector $\mathbf{n}$ (see Figure 2 ). Note that $\partial \mathrm{D}$ does not necessarily coincide with a physical boundary. For the moment, we assume that the medium parameters in both

Table 1. States for the unified reciprocity theorems.

\begin{tabular}{lcc} 
& State $A$ & State $B$ \\
\hline Wavefields & $\hat{\mathbf{u}}_{A}(\mathbf{x}, \omega)$ & $\hat{\mathbf{u}}_{B}(\mathbf{x}, \omega)$ \\
Medium parameters & $\left\{\hat{\mathbf{A}}_{A}, \hat{\mathbf{B}}_{A}\right\}(\mathbf{x}, \omega)$ & $\left\{\hat{\mathbf{A}}_{B}, \hat{\mathbf{B}}_{B}\right\}(\mathbf{x}, \omega)$ \\
Boundary parameters & $\hat{\mathbf{Y}}_{A}(\mathbf{x}, \omega)$ & $\hat{\mathbf{Y}}_{B}(\mathbf{x}, \omega)$ \\
Source functions & $\hat{\mathbf{s}}_{A}(\mathbf{x}, \omega)$ & $\hat{\mathbf{s}}_{B}(\mathbf{x}, \omega)$ \\
\hline
\end{tabular}

Domain D 
states are continuous in D. We integrate both sides of equation 22 over this domain and apply Gauss' theorem in matrix-vector form (equation A-5, see Appendix A) to the left-hand side. This yields

$$
\begin{aligned}
\oint_{\partial \mathrm{D}} \hat{\mathbf{u}}_{A}^{T} \mathbf{K} \mathbf{N}_{\mathbf{x}} \hat{\mathbf{u}}_{B} \mathrm{~d}^{2} \mathbf{x}= & \int_{\mathrm{D}}\left\{\hat{\mathbf{u}}_{A}^{T} \mathbf{K} \hat{\mathbf{s}}_{B}-\hat{\mathbf{s}}_{A}^{T} \mathbf{K} \hat{\mathbf{u}}_{B}\right\} \mathrm{d}^{3} \mathbf{x} \\
& -\int_{\mathrm{D}} \hat{\mathbf{u}}_{A}^{T} \mathbf{K}\left\{j \omega\left(\hat{\mathbf{A}}_{B}-\hat{\mathbf{A}}_{A}\right)\right. \\
& \left.+\left(\hat{\mathbf{B}}_{B}-\hat{\mathbf{B}}_{A}\right)\right\} \hat{\mathbf{u}}_{B} \mathrm{~d}^{3} \mathbf{x} .
\end{aligned}
$$

Here, $\mathbf{N}_{\mathbf{x}}$ is a matrix containing the components of normal vector $\mathbf{n}$, organized in the same way as matrix $\mathbf{D}_{\mathbf{x}}$. For example, for the acoustic situation $\mathbf{N}_{\mathbf{x}}$ is defined as

$$
\mathbf{N}_{\mathbf{x}}=\left(\begin{array}{cccc}
0 & n_{1} & n_{2} & n_{3} \\
n_{1} & 0 & 0 & 0 \\
n_{2} & 0 & 0 & 0 \\
n_{3} & 0 & 0 & 0
\end{array}\right)
$$

For all situations, $\mathbf{N}_{\mathbf{x}}$ obeys the symmetry relation

$$
\mathbf{K} \mathbf{N}_{\mathbf{x}} \mathbf{K}=-\mathbf{N}_{\mathbf{x}}=-\mathbf{N}_{\mathbf{x}}^{T}
$$

analogous to equation 12. Equation 23 is the unified convolutiontype reciprocity theorem for a domain $\mathrm{D}$ in which the medium parameters are continuous. It interrelates the wavefield quantities (contained in $\hat{\mathbf{u}}_{A}$ and $\hat{\mathbf{u}}_{B}$ ), the medium parameters (contained in $\hat{\mathbf{A}}_{A}, \hat{\mathbf{A}}_{B}$, $\hat{\mathbf{B}}_{A}$, and $\hat{\mathbf{B}}_{B}$ ) and the source functions (contained in $\hat{\mathbf{s}}_{A}$ and $\hat{\mathbf{s}}_{B}$ ) of states $A$ and $B$. The left-hand side is a boundary integral that contains a specific combination of the wavefield quantities of states $A$ and $B$ at the boundary of domain $\mathrm{D}$. The first integral on the right-hand side interrelates the wavefield quantities and the source functions in $\mathbb{D}$. The second integral on the right-hand side contains the differences between the medium parameters in both states; obviously, this integral vanishes when the medium parameters in both states are identical.

We now extend the reciprocity-theorem equation 23 for the situation in which $\mathrm{D}$ contains internal interfaces (or fractures) with imperfect coupling. To this end, we subdivide $\mathbb{D}$ into $M$ continuous regions, according to $\mathbb{D}=\mathbb{D}_{1} \cup \mathbb{D}_{2} \cdots \cup \mathbb{D}_{M}$, see Figure 3 . Region $\mathbb{D}_{m}$ is enclosed by surface $\partial \mathbb{D}_{m}$ with outward-pointing normal vector $\mathbf{n}_{m}$. The boundaries between these regions represent the imperfect internal interfaces. Note that each internal interface is part of two surfaces $\partial \mathrm{D}_{m}$, with opposite-pointing normal vectors $\mathbf{n}_{m}$, see Figure 3 .

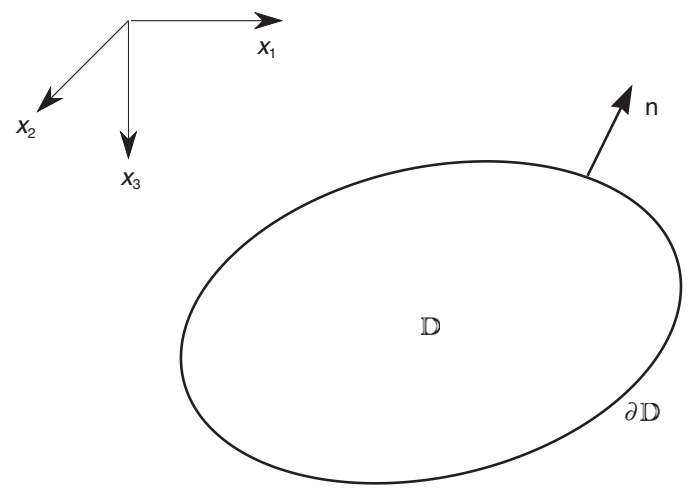

Figure 2. Configuration for the reciprocity theorems.
Since the medium parameters in region $\mathbb{D}_{m}$ are continuous, reciprocity-theorem equation 23 applies to each of these regions. Summing both sides of this equation over $m$ again yields equation 23 for the total domain $\mathbb{D}$, with an extra integral over the internal interfaces on the left-hand side as stated by

$$
\int_{\partial \mathrm{D}_{\text {int }}}\left(\left(\hat{\mathbf{u}}_{A}^{T} \mathbf{K} \mathbf{N}_{\mathbf{x}} \hat{\mathbf{u}}_{B}\right)_{1}+\left(\hat{\mathbf{u}}_{A}^{T} \mathbf{K} \mathbf{N}_{\mathbf{x}} \hat{\mathbf{u}}_{B}\right)_{2}\right) \mathrm{d}^{2} \mathbf{x},
$$

where $\partial \mathrm{D}_{\text {int }}$ constitutes the total of all internal interfaces; the subscripts 1 and 2 denote the two sides of the internal interfaces. Using the general boundary condition 14 for imperfect interfaces and the first of the symmetry relations in equation 19 , this internal interface integral can be rewritten as

$$
\int_{\partial \mathrm{D}_{\mathrm{int}}} \hat{\mathbf{u}}_{A}^{T} \mathbf{M}^{T} \mathbf{N}\left(\mathbf{I}-\hat{\mathbf{Z}}_{A}^{-1} \hat{\mathbf{Z}}_{B}\right) \mathbf{M} \hat{\mathbf{u}}_{B} \mathrm{~d}^{2} \mathbf{x}
$$

(Wapenaar et al., 2004) with

$$
\hat{\mathbf{Z}}=(\mathbf{I}+j \omega \hat{\mathbf{Y}} / 2)^{-1}(\mathbf{I}-j \omega \hat{\mathbf{Y}} / 2)
$$

$\hat{\mathbf{Z}}$ obeys the symmetry relation

$$
\hat{\mathbf{Z}}^{T} \mathbf{N}=\mathbf{N} \hat{\mathbf{Z}}^{-1}
$$

which follows from equations 19 and 28. For small $\hat{\mathbf{Y}}$, equation 27 simplifies to

$$
j \omega \int_{\partial \mathrm{D}_{\mathrm{int}}} \hat{\mathbf{u}}_{A}^{T} \mathbf{M}^{T} \mathbf{N}\left(\hat{\mathbf{Y}}_{B}-\hat{\mathbf{Y}}_{A}\right) \mathbf{M} \hat{\mathbf{u}}_{B} \mathrm{~d}^{2} \mathbf{x} .
$$

In the integrals in equations 27 and $30, \hat{\mathbf{u}}_{A}, \hat{\mathbf{u}}_{B}$, and $\mathbf{M}$ are all chosen at the same side of the interfaces (but which side is arbitrary).

Adding the internal interface integral of equation 27 to the lefthand side of equation 23 , we obtain

$$
\begin{aligned}
\oint_{\partial \mathrm{D}} & \hat{\mathbf{u}}_{A}^{T} \mathbf{K} \mathbf{N}_{\mathbf{x}} \hat{\mathbf{u}}_{B} \mathrm{~d}^{2} \mathbf{x}+\int_{\partial \mathrm{D}_{\mathrm{int}}} \hat{\mathbf{u}}_{A}^{T} \mathbf{M}^{T} \mathbf{N}\left(\mathbf{I}-\hat{\mathbf{Z}}_{A}^{-1} \hat{\mathbf{Z}}_{B}\right) \mathbf{M} \hat{\mathbf{u}}_{B} \mathrm{~d}^{2} \mathbf{x} \\
= & \int_{\mathrm{D}}\left\{\hat{\mathbf{u}}_{A}^{T} \mathbf{K} \hat{\mathbf{s}}_{B}-\hat{\mathbf{s}}_{A}^{T} \mathbf{K} \hat{\mathbf{u}}_{B}\right\} \mathrm{d}^{3} \mathbf{x}-\int_{\mathrm{D}} \hat{\mathbf{u}}_{A}^{T} \mathbf{K}\left\{j \omega\left(\hat{\mathbf{A}}_{B}-\hat{\mathbf{A}}_{A}\right)\right. \\
& \left.+\left(\hat{\mathbf{B}}_{B}-\hat{\mathbf{B}}_{A}\right)\right\} \hat{\mathbf{u}}_{B} \mathrm{~d}^{3} \mathbf{x}
\end{aligned}
$$

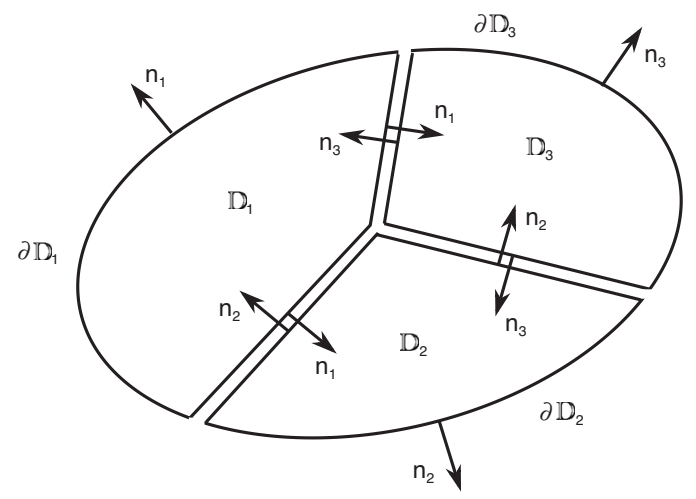

Figure 3. Piecewise continuous domain $\mathbb{D}=\mathbb{D}_{1} \cup \mathbb{D}_{2} \cdots \cup \mathbb{D}_{M}$. 
We conclude this section by specifying equation 31 for the acoustic situation, assuming small boundary parameters. Upon substitution of equations $2,3,13,17,20$, and 24 , we obtain

$$
\begin{aligned}
\oint_{\partial \mathrm{D}} & \left(\hat{p}_{A} \hat{v}_{i, B}-\hat{v}_{i, A} \hat{p}_{B}\right) n_{i} \mathrm{~d}^{2} \mathbf{x}+j \omega \int_{\partial \mathrm{D}_{\mathrm{int}}}\left\{\left(\hat{\kappa}_{B}^{\mathrm{b}}-\hat{\kappa}_{A}^{\mathrm{b}}\right) \hat{p}_{A} \hat{p}_{B}\right. \\
& \left.-\left(\hat{\rho}_{B}^{\mathrm{b}}-\hat{\rho}_{A}^{\mathrm{b}}\right) \hat{v}_{i, A} n_{i} \hat{v}_{j, B} n_{j}\right\} \mathrm{d}^{2} \mathbf{x} \\
= & \int_{\mathrm{D}}\left\{\hat{p}_{A} \hat{q}_{B}-\hat{v}_{i, A} \hat{f}_{i, B}-\hat{q}_{A} \hat{p}_{B}+\hat{f}_{i, A} \hat{v}_{i, B}\right\} \mathrm{d}^{3} \mathbf{x} \\
& -j \omega \int_{\mathrm{D}}\left\{\left(\hat{\kappa}_{B}-\hat{\kappa}_{A}\right) \hat{p}_{A} \hat{p}_{B}-\left(\hat{\rho}_{B}-\hat{\rho}_{A}\right) \hat{v}_{i, A} \hat{v}_{i, B}\right\} \mathrm{d}^{3} \mathbf{x} \\
& -\int_{\mathrm{D}}\left\{\left(\hat{b}_{B}^{p}-\hat{b}_{A}^{p}\right) \hat{p}_{A} \hat{p}_{B}-\left(\hat{b}_{B}^{v}-\hat{b}_{A}^{v}\right) \hat{v}_{i, A} \hat{v}_{i, B}\right\} \mathrm{d}^{3} \mathbf{x}, \quad
\end{aligned}
$$

which has the familiar form known from, e.g., Fokkema and van den Berg (1993) and de Hoop (1995), but with an extra integral over the internal interfaces.

\section{CORRELATION-TYPE RECIPROCITY THEOREM}

Porter (1970) and Bojarski (1983) formulated reciprocity theorems with back-propagating wavefields. Here, we extend these theorems for the general wave vector $\hat{\mathbf{u}}$. We consider the interaction quantity $\hat{\mathbf{u}}_{A}^{\dagger} \mathbf{D}_{\mathbf{x}} \hat{\mathbf{u}}_{B}+\left(\mathbf{D}_{\mathbf{x}} \hat{\mathbf{u}}_{A}\right)^{\dagger} \hat{\mathbf{u}}_{B}$. Since superscript $\dagger$ denotes transposition and complex conjugation, $\hat{\mathbf{u}}_{A}^{\dagger}$ represents a back-propagating wavefield. Using wave equation 9 as well as the symmetry relations in equations 10 and 11 for both states, we obtain

$$
\begin{aligned}
\hat{\mathbf{u}}_{A}^{\dagger} \mathbf{D}_{\mathbf{x}} \hat{\mathbf{u}}_{B}+\left(\mathbf{D}_{\mathbf{x}} \hat{\mathbf{u}}_{A}\right)^{\dagger} \hat{\mathbf{u}}_{B}= & \hat{\mathbf{u}}_{A}^{\dagger} \hat{\mathbf{s}}_{B}+\hat{\mathbf{s}}_{A}^{\dagger} \hat{\mathbf{u}}_{B}-\hat{\mathbf{u}}_{A}^{\dagger}\left\{j \omega\left(\hat{\mathbf{A}}_{B}-\hat{\mathbf{A}}_{A}^{*}\right)\right. \\
& \left.+\left(\hat{\mathbf{B}}_{B}+\hat{\mathbf{B}}_{A}^{\dagger}\right)\right\} \hat{\mathbf{u}}_{B} .
\end{aligned}
$$

This is the local form of the correlation-type matrix-vector reciprocity theorem. We say correlation type because the products in the frequency domain $\left(\hat{\mathbf{u}}_{A}^{\dagger} \hat{\mathbf{s}}_{B}\right.$ etc.) correspond to correlations in the time domain. Next, we consider again domain $\mathrm{D}$ with boundary $\partial \mathrm{D}$ and outward-pointing normal vector $\mathbf{n}$, see Figure 2. For the moment, we assume that the medium parameters in both states are continuous in $\mathrm{D}$. We integrate both sides of equation 33 over this domain and apply the theorem of Gauss in matrix-vector form (equation A-4) to the left-hand side. This yields

$$
\begin{aligned}
\oint_{\partial \mathrm{D}} \hat{\mathbf{u}}_{A}^{\dagger} \mathbf{N}_{\mathbf{x}} \hat{\mathbf{u}}_{B} \mathrm{~d}^{2} \mathbf{x}= & \int_{\mathrm{D}}\left\{\hat{\mathbf{u}}_{A}^{\dagger} \hat{\mathbf{s}}_{B}+\hat{\mathbf{s}}_{A}^{\dagger} \hat{\mathbf{u}}_{B}\right\} \mathrm{d}^{3} \mathbf{x} \\
& -\int_{\mathbb{D}} \hat{\mathbf{u}}_{A}^{\dagger}\left\{j \omega\left(\hat{\mathbf{A}}_{B}-\hat{\mathbf{A}}_{A}^{*}\right)\right. \\
& \left.+\left(\hat{\mathbf{B}}_{B}+\hat{\mathbf{B}}_{A}^{\dagger}\right)\right\} \hat{\mathbf{u}}_{B} \mathrm{~d}^{3} \mathbf{x}
\end{aligned}
$$

Equation 34 is the unified correlation-type reciprocity theorem for a domain $\mathbb{D}$ in which the medium parameters are continuous.

We now extend this reciprocity theorem for the situation in which D contains internal interfaces with imperfect coupling, see Figure 3. Since the medium parameters in region $\mathbb{D}_{m}$ are continuous, reciprocity theorem 34 applies to each of these regions. Summing both sides of this equation over $m$ again yields equation 34 for the total domain
D, with an extra integral over the internal interfaces on the left-hand side, as stated by

$$
\int_{\partial \mathrm{D}_{\mathrm{int}}}\left(\left(\hat{\mathbf{u}}_{A}^{\dagger} \mathbf{N}_{\mathbf{x}} \hat{\mathbf{u}}_{B}\right)_{1}+\left(\hat{\mathbf{u}}_{A}^{\dagger} \mathbf{N}_{\mathbf{x}} \hat{\mathbf{u}}_{B}\right)_{2}\right) \mathrm{d}^{2} \mathbf{x} .
$$

Using the general boundary condition 14 for imperfect interfaces and the second of the symmetry relations in equation 19 , this internal interface integral can be rewritten as

$$
\int_{\partial \mathrm{D}_{\mathrm{int}}} \hat{\mathbf{u}}_{A}^{\dagger} \mathbf{M}^{T} \mathbf{J}\left(\mathbf{I}-\left(\hat{\mathbf{Z}}_{A}^{\prime}\right)^{-1} \hat{\mathbf{Z}}_{B}\right) \mathbf{M} \hat{\mathbf{u}}_{B} \mathrm{~d}^{2} \mathbf{x}
$$

(Wapenaar et al., 2004), with

$$
\hat{\mathbf{Z}}^{\prime}=\left(\mathbf{I}+j \omega \hat{\mathbf{Y}}^{*} / 2\right)^{-1}\left(\mathbf{I}-j \omega \hat{\mathbf{Y}}^{*} / 2\right) .
$$

Note that $\hat{\mathbf{Z}}^{\prime}$ obeys the symmetry relation

$$
\hat{\mathbf{Z}}^{\dagger} \mathbf{J}=\mathbf{J}\left(\hat{\mathbf{Z}}^{\prime}\right)^{-1},
$$

which follows from equations 19 and 37 . For small $\hat{\mathbf{Y}}$, equation 36 simplifies to

$$
j \omega \int_{\partial \mathrm{D}_{\mathrm{int}}} \hat{\mathbf{u}}_{A}^{\dagger} \mathbf{M}^{T} \mathbf{J}\left(\hat{\mathbf{Y}}_{B}-\hat{\mathbf{Y}}_{A}^{*}\right) \mathbf{M} \hat{\mathbf{u}}_{B} \mathrm{~d}^{2} \mathbf{x} .
$$

Adding the internal interface integral of equation 36 to the left-hand side of equation 34 , we obtain

$$
\begin{aligned}
\oint_{\partial \mathrm{D}} & \hat{\mathbf{u}}_{A}^{\dagger} \mathbf{N}_{\mathbf{x}} \hat{\mathbf{u}}_{B} \mathrm{~d}^{2} \mathbf{x}+\int_{\partial \mathrm{D}_{\mathrm{int}}} \hat{\mathbf{u}}_{A}^{\dagger} \mathbf{M}^{T} \mathbf{J}\left(\mathbf{I}-\left(\hat{\mathbf{Z}}_{A}^{\prime}\right)^{-1} \hat{\mathbf{Z}}_{B}\right) \mathbf{M} \hat{\mathbf{u}}_{B} \mathrm{~d}^{2} \mathbf{x} \\
= & \int_{\mathrm{D}}\left\{\hat{\mathbf{u}}_{A}^{\dagger} \hat{\mathbf{s}}_{B}+\hat{\mathbf{s}}_{A}^{\dagger} \hat{\mathbf{u}}_{B}\right\} \mathrm{d}^{3} \mathbf{x}-\int_{\mathrm{D}} \hat{\mathbf{u}}_{A}^{\dagger}\left\{j \omega\left(\hat{\mathbf{A}}_{B}-\hat{\mathbf{A}}_{A}^{*}\right)\right. \\
& \left.+\left(\hat{\mathbf{B}}_{B}+\hat{\mathbf{B}}_{A}^{\dagger}\right)\right\} \hat{\mathbf{u}}_{B} \mathrm{~d}^{3} \mathbf{x} .
\end{aligned}
$$

Note that when the medium and boundary parameters, sources, and wavefields are identical in both states, this reciprocity theorem reduces (omitting the subscripts $A$ and $B$ ) to

$$
\begin{aligned}
2 \Re \int_{\mathbb{D}} \hat{\mathbf{u}}^{\dagger} \hat{\mathbf{s}} \mathrm{d}^{3} \mathbf{x}= & \oint_{\partial \mathrm{D}} \hat{\mathbf{u}}^{\dagger} \mathbf{N}_{\mathbf{x}} \hat{\mathbf{u}} \mathrm{d}^{2} \mathbf{x} \\
& +\int_{\mathrm{D}} \hat{\mathbf{u}}^{\dagger}\left\{-2 \omega \Im(\hat{\mathbf{A}})+\hat{\mathbf{B}}+\hat{\mathbf{B}}^{\dagger}\right\} \hat{\mathbf{u}} \mathrm{d}^{3} \mathbf{x} \\
& +\int_{\partial \mathrm{D}_{\mathrm{int}}} \hat{\mathbf{u}}^{\dagger} \mathbf{M}^{T} \mathbf{J}\left(\mathbf{I}-\left(\hat{\mathbf{Z}}^{\prime}\right)^{-1} \hat{\mathbf{Z}}\right) \mathbf{M} \hat{\mathbf{u}} \mathrm{d}^{2} \mathbf{x},
\end{aligned}
$$

where $\mathfrak{R}$ and $\mathfrak{I}$ denote the real and imaginary part, respectively. Note that this form of the reciprocity theorem represents a power balance for each of the wave phenomena treated in this paper. The term on the left-hand side represents the power generated by the sources in $\mathbb{D}$. The first term on the right-hand side represents the power-flux propagating outward through $\partial \mathbb{D}$, the second term the power dissipated by the medium in $\mathbb{D}$ (which vanishes for real-valued $\hat{\mathbf{A}}$ and zero $\hat{\mathbf{B}}$ ), and the last term the power dissipated by the internal imperfect interfaces $\partial \mathbb{D}_{\text {int }}$ (which vanishes for real-valued $\hat{\mathbf{Y}}$, as in the linear slip model of Schoenberg, 1980). 
We conclude this section by specifying equation 40 for the acoustic situation, assuming small boundary parameters. Upon substitution of equations $2,3,17,20$, and 24, we obtain

$$
\begin{aligned}
\oint_{\partial \mathrm{D}} & \left(\hat{p}_{A}^{*} \hat{v}_{i, B}+\hat{v}_{i, A}^{*} \hat{p}_{B}\right) n_{i} \mathrm{~d}^{2} \mathbf{x}+j \omega \int_{\partial \mathrm{D}_{\mathrm{int}}}\left\{\left(\hat{\kappa}_{B}^{\mathrm{b}}-\hat{\kappa}_{A}^{\mathrm{b} *}\right) \hat{p}_{A}^{*} \hat{p}_{B}\right. \\
& \left.+\left(\hat{\rho}_{B}^{\mathrm{b}}-\hat{\rho}_{A}^{\mathrm{b}}\right) \hat{v}_{i, A}^{*} n_{i} \hat{v}_{j, B} n_{j}\right\} \mathrm{d}^{2} \mathbf{x} \\
= & \int_{\mathrm{D}}\left\{\hat{p}_{A}^{*} \hat{q}_{B}+\hat{v}_{i, A}^{*} \hat{f}_{i, B}+\hat{q}_{A}^{*} \hat{p}_{B}+\hat{f}_{i, A}^{*} \hat{v}_{i, B}\right\} \mathrm{d}^{3} \mathbf{x} \\
& -j \omega \int_{\mathbb{D}}\left\{\left(\hat{\kappa}_{B}-\hat{\kappa}_{A}^{*}\right) \hat{p}_{A}^{*} \hat{p}_{B}+\left(\hat{\rho}_{B}-\hat{\rho}_{A}^{*}\right) \hat{v}_{i, A}^{*} \hat{v}_{i, B}\right\} \mathrm{d}^{3} \mathbf{x} \\
& -\int_{\mathrm{D}}\left\{\left(\hat{b}_{B}^{p}+\hat{b}_{A}^{p^{*}}\right) \hat{p}_{A}^{*} \hat{p}_{B}+\left(\hat{b}_{B}^{v}+\hat{b}_{A}^{v^{*}}\right) \hat{v}_{i, A}^{*} \hat{v}_{i, B}\right\} \mathrm{d}^{3} \mathbf{x},
\end{aligned}
$$

which has the familiar form known from, e.g., Fokkema and van den Berg (1993) and de Hoop (1995), but with an extra integral over the internal interfaces.

\section{GREEN'S MATRIX}

The wavefield vector $\hat{\mathbf{u}}(\mathbf{x}, \omega)$ and the source vector $\hat{\mathbf{s}}(\mathbf{x}, \omega)$ are $L$ $\times 1$ vectors, where the value of $L$ depends on the type of wavefield considered. A Green's function is defined as the wavefield that would be obtained if the source were an impulsive point source $\delta(\mathbf{x}$ $\left.-\mathbf{x}^{\prime}\right) \delta(t)$, or, in the frequency domain, a point source $\delta\left(\mathbf{x}-\mathbf{x}^{\prime}\right)$ with unit spectrum. Since the source vector $\hat{\mathbf{s}}$ contains $L$ different source functions, we may define $L$ different Green's wavefield vectors. We define the $l$ th Green's wavefield vector (with $1 \leq l \leq L$ ) as the causal solution of general wave equation 9 with boundary condition 14 , with source vector $\hat{\mathbf{s}}(\mathbf{x}, \omega)$ replaced by $\boldsymbol{i}_{l} \delta\left(\mathbf{x}-\mathbf{x}^{\prime}\right)$, where $\boldsymbol{i}_{l}$ is the $L$ $\times 1$ unit vector $(0, \cdots, 1, \cdots, 0)^{T}$, with ' 1 ' on the $l$ th position. Hence, in the space-frequency domain the $l$ th Green's wave vector obeys the relations

$$
j \omega \hat{\mathbf{A}} \hat{\mathbf{g}}_{l}+\hat{\mathbf{B}} \hat{\mathbf{g}}_{l}+\mathbf{D}_{\mathbf{x}} \hat{\mathbf{g}}_{l}=\mathbf{i}_{l} \delta\left(\mathbf{x}-\mathbf{x}^{\prime}\right)
$$

and

$$
\left[\mathbf{M} \hat{\mathbf{g}}_{l}\right]=-j \omega \hat{\mathbf{Y}}\left\langle\mathbf{M} \hat{\mathbf{g}}_{l}\right\rangle,
$$

where $\hat{\mathbf{g}}_{l}=\hat{\mathbf{g}}_{l}\left(\mathbf{x}, \mathbf{x}^{\prime}, \omega\right)$ is the $l$ th $L \times 1$ Green's wave vector observed at $\mathbf{x}$, due to a point source of the $l$ th type at $\mathbf{x}^{\prime}$. Due to their causal behaviour in the time domain, the components of these Green's vectors obey the Kramers-Kronig relations.

Equations 43 and 44 each represent $L$ matrix-vector equations for the $L$ Green's wave vectors $\hat{\mathbf{g}}_{l}$, with $1 \leq l \leq L$. For example, for the acoustic situation $(L=4)$, equation 43 reads

$$
\left(\begin{array}{cccc}
\hat{\eta} & \partial_{1} & \partial_{2} & \partial_{3} \\
\partial_{1} & \hat{\zeta} & 0 & 0 \\
\partial_{2} & 0 & \hat{\zeta} & 0 \\
\partial_{3} & 0 & 0 & \hat{\zeta}
\end{array}\right)\left(\begin{array}{c}
\hat{G}^{p, q}\left(\mathbf{x}, \mathbf{x}^{\prime}, \omega\right) \\
\hat{G}_{1}^{v, q}\left(\mathbf{x}, \mathbf{x}^{\prime}, \omega\right) \\
\hat{G}_{2}^{v, q}\left(\mathbf{x}, \mathbf{x}^{\prime}, \omega\right) \\
\hat{G}_{3}^{v, q}\left(\mathbf{x}, \mathbf{x}^{\prime}, \omega\right)
\end{array}\right)=\left(\begin{array}{c}
\delta\left(\mathbf{x}-\mathbf{x}^{\prime}\right) \\
0 \\
0 \\
0
\end{array}\right)
$$

for $l=1\left(\right.$ with $\hat{\eta}=j \omega \hat{\kappa}+\hat{b}^{p}$ and $\left.\hat{\zeta}=j \omega \hat{\rho}+\hat{b}^{v}\right)$,

$$
\left(\begin{array}{cccc}
\hat{\eta} & \partial_{1} & \partial_{2} & \partial_{3} \\
\partial_{1} & \hat{\zeta} & 0 & 0 \\
\partial_{2} & 0 & \hat{\zeta} & 0 \\
\partial_{3} & 0 & 0 & \hat{\zeta}
\end{array}\right)\left(\begin{array}{c}
\hat{G}_{, 1}^{p, f}\left(\mathbf{x}, \mathbf{x}^{\prime}, \omega\right) \\
\hat{G}_{1,1}^{v, f}\left(\mathbf{x}, \mathbf{x}^{\prime}, \omega\right) \\
\hat{G}_{2,1}^{v, f}\left(\mathbf{x}, \mathbf{x}^{\prime}, \omega\right) \\
\hat{G}_{3,1}^{v, f}\left(\mathbf{x}, \mathbf{x}^{\prime}, \omega\right)
\end{array}\right)=\left(\begin{array}{c}
0 \\
\delta\left(\mathbf{x}-\mathbf{x}^{\prime}\right) \\
0 \\
0
\end{array}\right)
$$

for $l=2$, etc. The superscripts of the Green's functions refer to the type of observed wavefield at $\mathbf{x}$ and the source type at $\mathbf{x}^{\prime}$, respectively; the subscripts denote the different components. We now combine the $L$ Green's vectors into a Green's matrix and the $L$ source vectors into a source matrix,

$$
\begin{aligned}
\left(\hat{\mathbf{g}}_{1} \ldots\right. & \left.\hat{\mathbf{g}}_{l} \ldots \hat{\mathbf{g}}_{L}\right)\left(\mathbf{x}, \mathbf{x}^{\prime}, \omega\right)=\hat{\mathbf{G}}\left(\mathbf{x}, \mathbf{x}^{\prime}, \omega\right), \\
\left(i_{1} \ldots i_{l} \ldots i_{L}\right) \delta\left(\mathbf{x}-\mathbf{x}^{\prime}\right) & =\mathbf{I} \delta\left(\mathbf{x}-\mathbf{x}^{\prime}\right),
\end{aligned}
$$

where $\hat{\mathbf{G}}\left(\mathbf{x}, \mathbf{x}^{\prime}, \omega\right)$ is the $L \times L$ Green's wavefield matrix and $\mathbf{I}$ is the $L \times L$ identity matrix. With this notation, equations 43 and 44 for $l$ $=1 \ldots L$ can be combined into

$$
j \omega \hat{\mathbf{A}} \hat{\mathbf{G}}+\hat{\mathbf{B}} \hat{\mathbf{G}}+\mathbf{D}_{\mathbf{x}} \hat{\mathbf{G}}=\mathbf{I} \delta\left(\mathbf{x}-\mathbf{x}^{\prime}\right)
$$

and

$$
[\mathbf{M} \hat{\mathbf{G}}]=-j \omega \hat{\mathbf{Y}}\langle\mathbf{M} \hat{\mathbf{G}}\rangle,
$$

respectively. These are the general wave equation and boundary condition for the Green's matrix $\hat{\mathbf{G}}\left(\mathbf{x}, \mathbf{x}^{\prime}, \omega\right)$.

Note that the wave vector $\hat{\mathbf{u}}(\mathbf{x}, \omega)$ and the Green's matrix $\hat{\mathbf{G}}\left(\mathbf{x}, \mathbf{x}^{\prime}, \omega\right)$ obey the same linear wave equation with the same linear boundary conditions, but with different source functions, $\hat{\mathbf{s}}(\mathbf{x}, \omega)$ and $\mathbf{I} \delta\left(\mathbf{x}-\mathbf{x}^{\prime}\right)$, respectively. Hence, we may apply the superposition principle to express the wave vector as

$$
\hat{\mathbf{u}}(\mathbf{x}, \omega)=\int_{\mathbb{D}_{s}} \hat{\mathbf{G}}\left(\mathbf{x}, \mathbf{x}^{\prime}, \omega\right) \hat{\mathbf{s}}\left(\mathbf{x}^{\prime}, \omega\right) \mathrm{d}^{3} \mathbf{x}^{\prime},
$$

where $\mathrm{D}_{s}$ is the domain occupied by the source distribution. In the following, we derive representations for the Green's matrix $\hat{\mathbf{G}}\left(\mathbf{x}, \mathbf{x}^{\prime}, \omega\right)$, and, implicitly via equation 51 , for the wave vector $\hat{\mathbf{u}}(\mathbf{x}, \omega)$.

\section{CONVOLUTION-TYPE REPRESENTATION}

We consider again the piecewise continuous domain $\mathbb{D}$ with boundary $\partial \mathrm{D}$ and outward-pointing normal vector $\mathbf{n}$ (Figure 3 ). We assume that the boundaries between the different regions are imperfect interfaces; the combination of all internal interfaces is represented by $\partial \mathrm{D}_{\text {int }}$.

We derive a convolution-type representation for the Green's matrix. To this end, we let the Green's matrix introduced in the previous section, with the point source at $\mathbf{x}=\mathbf{x}^{\prime}$, play the role of state $A$ in the unified reciprocity theorem of equation 31 . For the medium parameters as well as for the boundary parameters, we introduce background values; hence, the Green's matrix in state $A$ is defined in a background medium. This is denoted by bars above the parameter matrices as well as the Green's matrix, see Table 2. A similar Green's matrix, but with its point source at $\mathbf{x}=\mathbf{x}^{\prime \prime}$, will play the role of state 
$B$. The medium parameters in this state are the actual parameters, and, consequently, the Green's matrix in state $B$ is defined in the actual medium, again see Table 2 .

Consider the following property of the delta function

$$
\int_{\mathbb{D}} \delta\left(\mathbf{x}-\mathbf{x}^{\prime}\right) u(\mathbf{x}) \mathrm{d}^{3} \mathbf{x}=\chi_{\mathbb{D}}\left(\mathbf{x}^{\prime}\right) u\left(\mathbf{x}^{\prime}\right)
$$

where $\chi_{\mathrm{D}}\left(\mathbf{x}^{\prime}\right)$ is the characteristic function for domain $\mathrm{D}$, defined as

$$
\chi_{\mathbb{D}}\left(\mathbf{x}^{\prime}\right)= \begin{cases}1 & \text { for } \mathbf{x}^{\prime} \in \mathbb{D} \\ \frac{1}{2} & \text { for } \mathbf{x}^{\prime} \in \partial \mathbb{D} \\ 0 & \text { for } \mathbf{x}^{\prime} \in \mathbb{R}^{3} \backslash\{\mathbb{D} \cup \partial \mathbb{D}\} .\end{cases}
$$

Upon substitution of the states of Table 2 into the convolution-type reciprocity theorem (equation 31 ), using this property of the delta function, we obtain

$$
\begin{aligned}
& \chi_{\mathrm{D}}\left(\mathbf{x}^{\prime \prime}\right) \mathbf{K} \hat{\overline{\mathbf{G}}}^{T}\left(\mathbf{x}^{\prime \prime}, \mathbf{x}^{\prime}, \omega\right) \mathbf{K}-\chi_{\mathrm{D}}\left(\mathbf{x}^{\prime}\right) \hat{\mathbf{G}}\left(\mathbf{x}^{\prime}, \mathbf{x}^{\prime \prime}, \omega\right) \\
& =\oint_{\partial \mathrm{D}} \mathbf{K} \hat{\overline{\mathbf{G}}}^{T}\left(\mathbf{x}, \mathbf{x}^{\prime}, \omega\right) \mathbf{K} \mathbf{N}_{\mathbf{x}} \hat{\mathbf{G}}\left(\mathbf{x}, \mathbf{x}^{\prime \prime}, \omega\right) \mathrm{d}^{2} \mathbf{x} \\
& \quad+\int_{\mathrm{D}} \mathbf{K} \hat{\overline{\mathbf{G}}}^{T}\left(\mathbf{x}, \mathbf{x}^{\prime}, \omega\right) \mathbf{K} \Delta \hat{\mathbf{H}}(\mathbf{x}, \omega) \hat{\mathbf{G}}\left(\mathbf{x}, \mathbf{x}^{\prime \prime}, \omega\right) \mathrm{d}^{3} \mathbf{x} \\
& \quad+\int_{\partial \mathrm{D}_{\mathrm{int}}} \mathbf{K} \hat{\overline{\mathbf{G}}}^{T}\left(\mathbf{x}, \mathbf{x}^{\prime}, \omega\right) \mathbf{K} \Delta \hat{\mathbf{H}}^{\mathrm{b}}(\mathbf{x}, \omega) \hat{\mathbf{G}}\left(\mathbf{x}, \mathbf{x}^{\prime \prime}, \omega\right) \mathrm{d}^{2} \mathbf{x}
\end{aligned}
$$

with the contrast functions $\Delta \hat{\mathbf{H}}$ and $\Delta \hat{\mathbf{H}}^{\mathrm{b}}$ defined as

$$
\begin{aligned}
\Delta \hat{\mathbf{H}} & =j \omega(\hat{\mathbf{A}}-\hat{\overline{\mathbf{A}}})+(\hat{\mathbf{B}}-\hat{\overline{\mathbf{B}}}), \\
\Delta \hat{\mathbf{H}}^{\mathrm{b}} & =\mathbf{K} \mathbf{M}^{T} \mathbf{N}\left\{\mathbf{I}-\hat{\overline{\mathbf{Z}}}^{-1} \hat{\mathbf{Z}}\right\} \mathbf{M} .
\end{aligned}
$$

Equation 54 is the general convolution-type representation of the Green's matrix. Applications are discussed in a later section. Here, we derive a reciprocity relation for the Green's matrix. To this end we replace the background parameters in state $A$ by the actual parameters; hence, the last two integrals on the right-hand side of equation 54 vanish. Then, we replace $D$ by $\mathbb{R}^{3}$, to make the characteristic functions in the left-hand side of equation 54 both equal 1. Finally, we assume that outside some sphere with finite radius, the medium is homogeneous, isotropic, and nonporous, which implies that the first integral on the right-hand side vanishes as well (Sommerfeld radiation conditions, Born and Wolf, 1965; Pao and Varatharajulu, 1976; de Hoop, 1995). This leaves

$$
\mathbf{K} \hat{\mathbf{G}}^{T}\left(\mathbf{x}^{\prime \prime}, \mathbf{x}^{\prime}, \omega\right) \mathbf{K}=\hat{\mathbf{G}}\left(\mathbf{x}^{\prime}, \mathbf{x}^{\prime \prime}, \omega\right)
$$

Of course a similar relation holds for the Green's matrix in the background medium. Equation 57 formulates source-receiver reciprocity for a piecewise continuous medium with imperfect interfaces. For example, for the acoustic situation, we have

$$
\begin{aligned}
\left(\begin{array}{cccc}
\hat{G}^{p, q} & -\hat{G}_{1}^{v, q} & -\hat{G}_{2}^{v, q} & -\hat{G}_{3}^{v, q} \\
-\hat{G}_{, 1}^{p, f} & \hat{G}_{1,1}^{v, f} & \hat{G}_{2,1}^{v, f} & \hat{G}_{3,1}^{v, f} \\
-\hat{G}_{, 2}^{p, f} & \hat{G}_{1,2}^{v, f} & \hat{G}_{2,2}^{v, f} & \hat{G}_{3,2}^{v, f} \\
-\hat{G}_{, 3}^{p, f} & \hat{G}_{1,3}^{v, f} & \hat{G}_{2,3}^{v, f} & \hat{G}_{3,3}^{v, f}
\end{array}\right)\left(\mathbf{x}^{\prime \prime}, \mathbf{x}^{\prime}, \omega\right) \\
= \\
=\left(\begin{array}{cccc}
\hat{G}^{p, q} & \hat{G}_{, 1}^{p, f} & \hat{G}_{, 2}^{p, f} & \hat{G}_{, 3}^{p, f} \\
\hat{G}_{1}^{v, q} & \hat{G}_{1,1}^{v, f} & \hat{G}_{1,2}^{v, f} & \hat{G}_{1,3}^{v, f} \\
\hat{G}_{2}^{v, q} & \hat{G}_{2,1}^{v, f} & \hat{G}_{2,2}^{v, f} & \hat{G}_{2,3}^{v, f} \\
\hat{G}_{3}^{v, q} & \hat{G}_{3,1}^{v, f} & \hat{G}_{3,2}^{v, f} & \hat{G}_{3,3}^{v, f}
\end{array}\right)\left(\mathbf{x}^{\prime}, \mathbf{x}^{\prime \prime}, \omega\right) .
\end{aligned}
$$

\section{CORRELATION-TYPE REPRESENTATION}

For the derivation of a correlation-type representation of the Green's matrix, we substitute the states of Table 2 into the reciprocity theorem of correlation-type (equation 40), which gives

$$
\begin{aligned}
\chi_{\mathrm{D}}\left(\mathbf{x}^{\prime \prime}\right) \hat{\overline{\mathbf{G}}}^{\dagger}\left(\mathbf{x}^{\prime \prime}, \mathbf{x}^{\prime}, \omega\right)+\chi_{\mathrm{D}}\left(\mathbf{x}^{\prime}\right) \hat{\mathbf{G}}\left(\mathbf{x}^{\prime}, \mathbf{x}^{\prime \prime}, \omega\right) \\
=\oint_{\partial \mathrm{D}} \hat{\overline{\mathbf{G}}}^{\dagger}\left(\mathbf{x}, \mathbf{x}^{\prime}, \omega\right) \mathbf{N}_{\mathbf{x}} \hat{\mathbf{G}}\left(\mathbf{x}, \mathbf{x}^{\prime \prime}, \omega\right) \mathrm{d}^{2} \mathbf{x} \\
\quad+\int_{\mathrm{D}} \hat{\overline{\mathbf{G}}}^{\dagger}\left(\mathbf{x}, \mathbf{x}^{\prime}, \omega\right) \Delta \hat{\mathbf{H}}(\mathbf{x}, \omega) \hat{\mathbf{G}}\left(\mathbf{x}, \mathbf{x}^{\prime \prime}, \omega\right) \mathrm{d}^{3} \mathbf{x} \\
\quad+\int_{\partial \mathrm{D}_{\text {int }}} \hat{\overline{\mathbf{G}}}^{\dagger}\left(\mathbf{x}, \mathbf{x}^{\prime}, \omega\right) \Delta \hat{\mathbf{H}}^{\mathrm{b}}(\mathbf{x}, \omega) \hat{\mathbf{G}}\left(\mathbf{x}, \mathbf{x}^{\prime \prime}, \omega\right) \mathrm{d}^{2} \mathbf{x},
\end{aligned}
$$

with the contrast functions $\Delta \hat{\mathbf{H}}$ and $\Delta \hat{\mathbf{H}}^{\mathrm{b}}$ now defined as

$$
\begin{aligned}
\Delta \hat{\mathbf{H}} & =j \omega\left(\hat{\mathbf{A}}-\hat{\overline{\mathbf{A}}}^{*}\right)+\left(\hat{\mathbf{B}}+\hat{\overline{\mathbf{B}}}^{\dagger}\right), \\
\Delta \hat{\mathbf{H}}^{\mathrm{b}} & =\mathbf{M}^{T} \mathbf{J}\left\{\mathbf{I}-\left(\hat{\overline{\mathbf{Z}}}^{\prime}\right)^{-1} \hat{\mathbf{Z}}\right\} \mathbf{M} .
\end{aligned}
$$

Equation 59 is the general correlation-type representation of the Green's matrix.

\section{APPLICATIONS}

Here we discuss a number of applications of the general convolution-type and correlation-type representations. This overview is not exhaustive but serves as an illustration.

Table 2. Green's states for the unified representations.

\begin{tabular}{lcc}
\hline & State $A$ & State $B$ \\
\hline Wavefields & $\hat{\overline{\mathbf{G}}}\left(\mathbf{x}, \mathbf{x}^{\prime}, \omega\right)$ & $\hat{\mathbf{G}}\left(\mathbf{x}, \mathbf{x}^{\prime \prime}, \omega\right)$ \\
Medium parameters & $\hat{\overline{\mathbf{A}}}, \hat{\overline{\mathbf{B}}}\}(\mathbf{x}, \omega)$ & $\{\hat{\mathbf{A}}, \hat{\mathbf{B}}\}(\mathbf{x}, \omega)$ \\
Boundary parameters & $\hat{\overline{\mathbf{Y}}}(\mathbf{x}, \omega)$ & $\hat{\mathbf{Y}}(\mathbf{x}, \omega)$ \\
Source functions & $\mathbf{I} \delta\left(\mathbf{x}-\mathbf{x}^{\prime}\right)$ & $\mathbf{I} \delta\left(\mathbf{x}-\mathbf{x}^{\prime \prime}\right)$ \\
\hline & Domain $\mathrm{D}$ & \\
\hline
\end{tabular}




\section{Forward wavefield extrapolation}

The most straightforward application of the convolution-type representation (equation 54) is forward wavefield extrapolation. Consider the configuration of Figure 4 . The boundary $\partial \mathrm{D}$ consists of an acquisition surface $\partial \mathrm{D}_{1}$ and a hemisphere $\partial \mathrm{D}_{0}$ in the upper half-space with its midpoint at $\mathbf{x}^{\prime}$. The source domain $\mathrm{D}_{s}$ is sited below the acquisition surface $\partial \mathrm{D}_{1}$. When we let the radius of the hemisphere go to infinity and assume that beyond some finite radius the medium is homogeneous, isotropic, and nonporous, the contribution of the boundary integral over $\partial \mathrm{D}_{0}$ vanishes. Assuming the contrasts $\Delta \hat{\mathbf{H}}$ and $\Delta \hat{\mathbf{H}}^{\mathrm{b}}$ defined by equations 55 and 56 are negligible in $\mathbb{D}$, we obtain from equation 54 (using equation 57)

$\hat{\mathbf{G}}\left(\mathbf{x}^{\prime}, \mathbf{x}^{\prime \prime}, \omega\right)=-\int_{\partial \mathrm{D}_{1}} \hat{\overline{\mathbf{G}}}\left(\mathbf{x}^{\prime}, \mathbf{x}, \omega\right) \mathbf{N}_{\mathbf{x}} \hat{\mathbf{G}}\left(\mathbf{x}, \mathbf{x}^{\prime \prime}, \omega\right) \mathrm{d}^{2} \mathbf{x}$.

Multiplying both sides by $\hat{\mathbf{s}}\left(\mathbf{x}^{\prime \prime}, \omega\right)$ and integrating over the source domain $\mathbb{D}_{s}$, we obtain (using equation 51)

$$
\hat{\mathbf{u}}\left(\mathbf{x}^{\prime}, \omega\right)=-\int_{\partial \mathrm{D}_{1}} \hat{\overline{\mathbf{G}}}\left(\mathbf{x}^{\prime}, \mathbf{x}, \omega\right) \mathbf{N}_{\mathbf{x}} \hat{\mathbf{u}}(\mathbf{x}, \omega) \mathrm{d}^{2} \mathbf{x} .
$$

This expression formulates forward extrapolation of the wavefield $\hat{\mathbf{u}}(\mathbf{x}, \omega)$ at acquisition surface $\partial \mathrm{D}_{1}$, because of sources below this surface, to any point $\mathbf{x}^{\prime}$ above this surface. By substituting the vectors and matrices for the acoustic situation, we obtain

$$
\begin{aligned}
\hat{p}\left(\mathbf{x}^{\prime}, \omega\right)= & -\int_{\partial \mathrm{D}_{1}}\left\{\hat{\bar{G}}^{p, q}\left(\mathbf{x}^{\prime}, \mathbf{x}, \omega\right) \hat{v}_{j}(\mathbf{x}, \omega)\right. \\
& \left.+\hat{\bar{G}}_{, j}^{p, f}\left(\mathbf{x}^{\prime}, \mathbf{x}, \omega\right) \hat{p}(\mathbf{x}, \omega)\right\} n_{j} \mathrm{~d}^{2} \mathbf{x}, \\
\hat{v}_{i}\left(\mathbf{x}^{\prime}, \omega\right)= & -\int_{\partial \mathrm{D}_{1}}\left\{\hat{\bar{G}}_{i}^{v, q}\left(\mathbf{x}^{\prime}, \mathbf{x}, \omega\right) \hat{v}_{j}(\mathbf{x}, \omega)\right. \\
& \left.+\hat{\bar{G}}_{i, j}^{v, f}\left(\mathbf{x}^{\prime}, \mathbf{x}, \omega\right) \hat{p}(\mathbf{x}, \omega)\right\} n_{j} \mathrm{~d}^{2} \mathbf{x},
\end{aligned}
$$

which are the well-known Kirchhoff-Helmholtz integrals (Morse and Feshbach, 1953; Berkhout, 1985), with many applications in

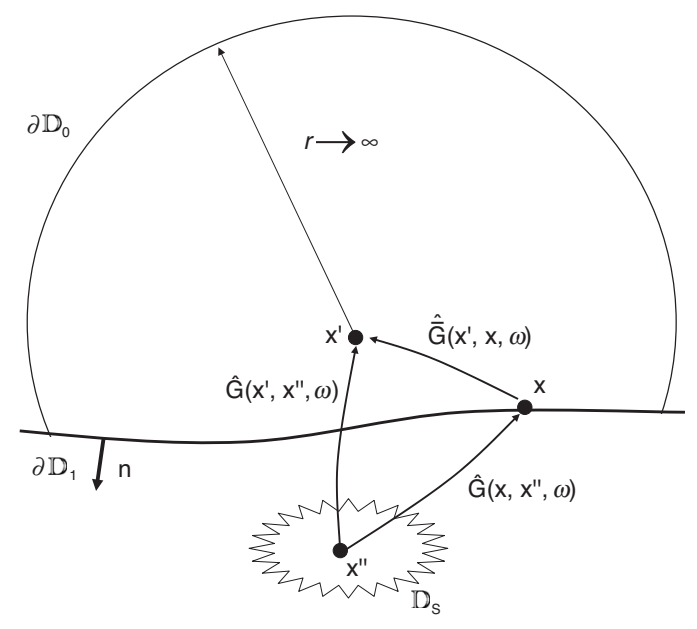

Figure 4. Configuration for forward wavefield extrapolation. seismic modeling (Frazer and Sen, 1985; Hill and Wuenschel, 1985; Wenzel et al., 1990; Druzhinin et al., 1998). Equation 63 is the generalization of the Kirchhoff-Helmholtz integral for any of the wave phenomena considered in this paper.

\section{Inverse wavefield extrapolation}

An expression for inverse wavefield extrapolation follows in a similar way from the correlation-type representation in equation 59. Consider the configuration of Figure 5. The boundary $\partial \mathbb{D}$ now consists of an acquisition surface $\partial \mathrm{D}_{0}$, a horizontal surface $\partial \mathrm{D}_{1}$ between $\mathbf{x}^{\prime}$ and the source domain $\mathrm{D}_{s}$, and a cylindrical surface $\partial \mathrm{D}_{\text {cyl }}$ with a vertical axis through $\mathbf{x}^{\prime}$ (Figure 5 is a side-view of this configuration). When we let the radius of this cylindrical surface go to infinity, the contribution of the boundary integral over $\partial \mathrm{D}_{\text {cyl }}$ vanishes for body waves. The boundary integral over $\partial \mathrm{D}_{1}$ contains an evanescent wave contribution and a contribution proportional to the square of the reflection coefficients of the interfaces in domain $\mathrm{D}$ (Wapenaar and Berkhout, 1989). Ignoring these contributions and assuming that the medium and interfaces in $\mathrm{D}$ are lossless and the contrasts $\Delta \hat{\mathbf{H}}$ and $\Delta \hat{\mathbf{H}}^{\mathrm{b}}$ defined by equations 60 and 61 are negligible in $\mathrm{D}$, we obtain from equation 59 (using equation 57)

$\hat{\mathbf{G}}\left(\mathbf{x}^{\prime}, \mathbf{x}^{\prime \prime}, \omega\right)=-\int_{\partial \mathrm{D}_{0}} \mathbf{K} \hat{\overline{\mathbf{G}}}^{*}\left(\mathbf{x}^{\prime}, \mathbf{x}, \omega\right) \mathbf{K} \mathbf{N}_{\mathbf{x}} \hat{\mathbf{G}}\left(\mathbf{x}, \mathbf{x}^{\prime \prime}, \omega\right) \mathrm{d}^{2} \mathbf{x}$,

or, using equation 51 ,

$$
\hat{\mathbf{u}}\left(\mathbf{x}^{\prime}, \omega\right)=-\int_{\partial \mathrm{D}_{0}} \mathbf{K} \hat{\overline{\mathbf{G}}}^{*}\left(\mathbf{x}^{\prime}, \mathbf{x}, \omega\right) \mathbf{K N}_{\mathbf{x}} \hat{\mathbf{u}}(\mathbf{x}, \omega) \mathrm{d}^{2} \mathbf{x} .
$$

This expression formulates inverse extrapolation of the wavefield $\hat{\mathbf{u}}(\mathbf{x}, \omega)$ at acquisition surface $\partial \mathbb{D}_{0}$, because of sources below this surface, to any point $\mathbf{x}^{\prime}$ between this surface and the sources. It is a generalization of the Kirchhoff-Helmholtz integral for inverse wavefield extrapolation (Schneider, 1978; Berkhout, 1985; Bleistein, 1987; Tygel et al., 2000), with applications in seismic migration. Unlike in the derivation of equation 63 for forward extrapolation, we assumed that the medium and interfaces in $\mathbb{D}$ are lossless. When the medium and/or interfaces in $\mathrm{D}$ are not lossless, we can choose the reference parameters as follows: $\hat{\overline{\mathbf{A}}}=\hat{\mathbf{A}}^{*}, \hat{\overline{\mathbf{B}}}=-\hat{\mathbf{B}}^{\dagger}$, and $\hat{\overline{\mathbf{Y}}}=\hat{\mathbf{Y}}^{*}$. As a consequence, the contrast functions $\Delta \hat{\mathbf{H}}$ and $\Delta \hat{\mathbf{H}}^{\mathrm{b}}$ defined by equations 60 and 61 are zero, so equations 66 and 67 remain valid. However, this choice of reference parameters implies that the Green's function $\hat{\overline{\mathbf{G}}}\left(\mathbf{x}^{\prime}, \mathbf{x}, \omega\right)$ propagating through the reference medium is

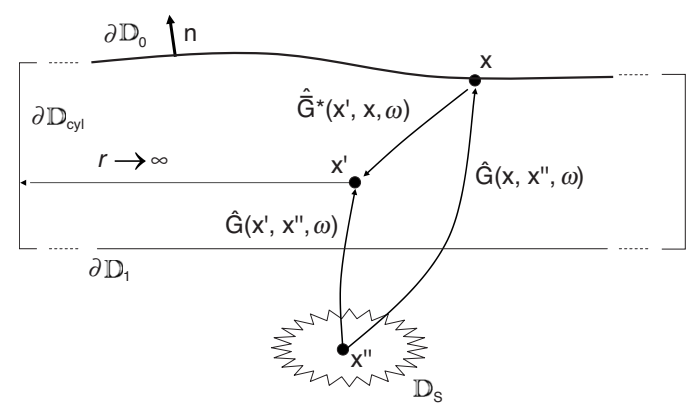

Figure 5. Configuration for inverse wavefield extrapolation. 
growing exponentially (to compensate for the exponential decay in the wavefield $\hat{\mathbf{u}}(\mathbf{x}, \omega))$. Hence, the implementation of the inverse extrapolation integral for attenuating media should be done with utmost care to avoid instabilities (Mittet et al., 1995; Zhang and Wapenaar, 2002).

\section{Boundary integral representation (perfect interfaces)}

We derive a representation for the scattered wavefield above a perfectly coupled interface. Consider the configuration of Figure 6, in which $\partial \mathbb{D}_{1}$ represents an interface. Assuming the contrasts $\Delta \hat{\mathbf{H}}$ and $\Delta \hat{\mathbf{H}}^{\text {b }}$ defined by equations 55 and 56 are negligible in $\mathrm{D}$, we obtain from equation 54 (using equation 57)

$$
\begin{aligned}
\hat{\mathbf{G}}\left(\mathbf{x}^{\prime}, \mathbf{x}^{\prime \prime}, \omega\right)= & \hat{\overline{\mathbf{G}}}\left(\mathbf{x}^{\prime}, \mathbf{x}^{\prime \prime}, \omega\right) \\
& -\int_{\partial \mathrm{D}_{1}} \hat{\overline{\mathbf{G}}}\left(\mathbf{x}^{\prime}, \mathbf{x}, \omega\right) \mathbf{N}_{\mathbf{x}} \hat{\mathbf{G}}\left(\mathbf{x}, \mathbf{x}^{\prime \prime}, \omega\right) \mathrm{d}^{2} \mathbf{x} .
\end{aligned}
$$

We define the Green's matrix as a superposition of an incident and a scattered contribution, as stated by

$$
\hat{\mathbf{G}}\left(\mathbf{x}, \mathbf{x}^{\prime \prime}, \omega\right)=\hat{\mathbf{G}}^{\text {inc }}\left(\mathbf{x}, \mathbf{x}^{\prime \prime}, \omega\right)+\hat{\mathbf{G}}^{\mathrm{sct}}\left(\mathbf{x}, \mathbf{x}^{\prime \prime}, \omega\right),
$$

where $\hat{\mathbf{G}}^{\text {inc }}\left(\mathbf{x}, \mathbf{x}^{\prime \prime}, \omega\right)=\hat{\overline{\mathbf{G}}}\left(\mathbf{x}, \mathbf{x}^{\prime \prime}, \omega\right)$. Equation 68 remains valid when outside D, i.e., in the lower half-space, the actual and reference medium parameters are different. We choose the reference parameters in the lower half-space such that they are continuous across $\partial \mathbb{D}_{1}$, and homogeneous, isotropic, and nonporous beyond some finite domain in the lower half-space. Consequently,

$$
\int_{\partial \mathrm{D}_{1}} \hat{\overline{\mathbf{G}}}\left(\mathbf{x}^{\prime}, \mathbf{x}, \omega\right) \mathbf{N}_{\mathbf{x}} \hat{\mathbf{G}}^{\mathrm{inc}}\left(\mathbf{x}, \mathbf{x}^{\prime \prime}, \omega\right) \mathrm{d}^{2} \mathbf{x}=\mathbf{O}
$$

hence,

$\hat{\mathbf{G}}^{\mathrm{sct}}\left(\mathbf{x}^{\prime}, \mathbf{x}^{\prime \prime}, \omega\right)=-\int_{\partial \mathrm{D}_{1}} \hat{\overline{\mathbf{G}}}\left(\mathbf{x}^{\prime}, \mathbf{x}, \omega\right) \mathbf{N}_{\mathbf{x}} \hat{\mathbf{G}}^{\mathrm{sct}}\left(\mathbf{x}, \mathbf{x}^{\prime \prime}, \omega\right) \mathrm{d}^{2} \mathbf{x}$

or, using equation 51 ,

$$
\hat{\mathbf{u}}^{\mathrm{sct}}\left(\mathbf{x}^{\prime}, \omega\right)=-\int_{\partial \mathrm{D}_{1}} \hat{\overline{\mathbf{G}}}\left(\mathbf{x}^{\prime}, \mathbf{x}, \omega\right) \mathbf{N}_{\mathbf{x}} \hat{\mathbf{u}}^{\mathrm{sct}}(\mathbf{x}, \omega) \mathrm{d}^{2} \mathbf{x} .
$$

Note the analogy with equation 63 for forward wavefield extrapolation. The main difference is that $\hat{\mathbf{u}}$ in equation 63 is the upgoing wavefield because of sources below $\partial \mathrm{D}_{1}$, whereas $\hat{\mathbf{u}}^{\text {stt }}$ in equation 72 is the upgoing scattered wavefield at interface $\partial \mathrm{D}_{1}$ because of sources above this interface. This scattered wavefield can be expressed in terms of a reflection operator acting on the incident wavefield at $\partial \mathrm{D}_{1}$. For example, for the acoustic situation, it can be written as $\mathbf{N}_{\mathbf{x}} \hat{\mathbf{u}}^{\text {sct }}(\mathbf{x}, \omega) \approx-R(\mathbf{x}, \alpha) \mathbf{K} \mathbf{N}_{\mathbf{x}} \hat{\mathbf{u}}^{\text {inc }}(\mathbf{x}, \omega)$, where $R(\mathbf{x}, \alpha)$ is the local angle-dependent reflection coefficient. This is a generalization of what is commonly known as the Kirchhoff approximation (Bleistein, 1984).

\section{Boundary integral representation (imperfect interfaces)}

The derivation for the scattered wavefield above an imperfect interface is somewhat different. The interface is now represented by $\partial \mathbb{D}_{\text {int }}$, whereas $\partial \mathbb{D}$ is a sphere with infinite radius. From equation 54 , we thus obtain (using equation 57)

$$
\begin{aligned}
\hat{\mathbf{G}}\left(\mathbf{x}^{\prime}, \mathbf{x}^{\prime \prime}, \omega\right)= & \hat{\overline{\mathbf{G}}}\left(\mathbf{x}^{\prime}, \mathbf{x}^{\prime \prime}, \omega\right) \\
& -\int_{\partial \mathrm{D}_{\text {int }}} \hat{\overline{\mathbf{G}}}\left(\mathbf{x}^{\prime}, \mathbf{x}, \omega\right) \Delta \hat{\mathbf{H}}^{\mathrm{b}}(\mathbf{x}, \omega) \hat{\mathbf{G}}\left(\mathbf{x}, \mathbf{x}^{\prime \prime}, \omega\right) \mathrm{d}^{2} \mathbf{x},
\end{aligned}
$$

with $\Delta \hat{\mathbf{H}}^{\mathrm{b}}$ defined by equation 56 . Various choices are possible for the reference medium. Let us choose a reference medium that is identical to the actual medium, except that it has an interface with perfect coupling, i.e., $\hat{\overline{\mathbf{Y}}}=\mathbf{O}$, and hence $\hat{\overline{\mathbf{Z}}}=\mathbf{I}$. For $\Delta \hat{\mathbf{H}}^{\text {b }}$, we thus obtain (assuming small $\hat{\mathbf{Y}}$ )

$$
\Delta \hat{\mathbf{H}}^{\mathrm{b}}=\mathbf{K} \mathbf{M}^{T} \mathbf{N}\{\mathbf{I}-\hat{\mathbf{Z}}\} \mathbf{M} \approx j \omega \mathbf{K} \mathbf{M}^{T} \mathbf{N} \hat{\mathbf{Y}} \mathbf{M} .
$$

Moreover, for this choice, the reference Green's function $\hat{\overline{\mathbf{G}}}\left(\mathbf{x}^{\prime}, \mathbf{x}^{\prime \prime}, \omega\right)$ in equation 73 is equal to the actual Green's function $\hat{\mathbf{G}}\left(\mathbf{x}^{\prime}, \mathbf{x}^{\prime \prime}, \omega\right)$ in equation 68 .

Equation 73 is an integral equation of the second kind for $\hat{\mathbf{G}}\left(\mathbf{x}^{\prime}, \mathbf{x}^{\prime \prime}, \omega\right)$. It can be solved iteratively, according to

$$
\begin{aligned}
\left\{\hat{\mathbf{G}}\left(\mathbf{x}^{\prime}, \mathbf{x}^{\prime \prime}, \omega\right)\right\}^{(k)}= & \hat{\overline{\mathbf{G}}}\left(\mathbf{x}^{\prime}, \mathbf{x}^{\prime \prime}, \omega\right) \\
& -\int_{\partial \mathrm{D}_{\mathrm{int}}} \hat{\overline{\mathbf{G}}}\left(\mathbf{x}^{\prime}, \mathbf{x}, \omega\right) \Delta \hat{\mathbf{H}}^{\mathrm{b}}(\mathbf{x}, \omega) \\
& \times\left\{\hat{\mathbf{G}}\left(\mathbf{x}, \mathbf{x}^{\prime \prime}, \omega\right)\right\}^{(k-1)} \mathrm{d}^{2} \mathbf{x},
\end{aligned}
$$

for $k \geq 1$, with

$$
\left\{\hat{\mathbf{G}}\left(\mathbf{x}^{\prime}, \mathbf{x}^{\prime \prime}, \omega\right)\right\}^{(0)}=\hat{\overline{\mathbf{G}}}\left(\mathbf{x}^{\prime}, \mathbf{x}^{\prime \prime}, \omega\right) .
$$

\section{Volume integral representation}

The derivation for a volume integral representation is similar to that for the imperfect interfaces, but instead of the contrast function $\Delta \hat{\mathbf{H}}^{\mathrm{b}}$ at the internal interfaces, we consider the contrast function $\Delta \hat{\mathbf{H}}$

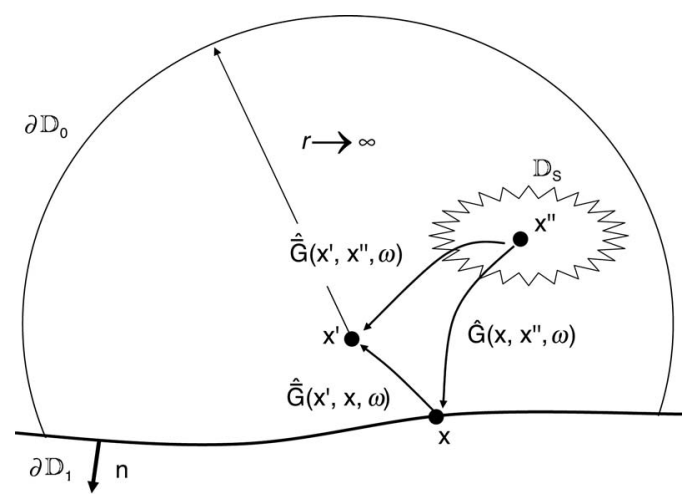

Figure 6. Configuration for boundary integral representation. 
in $\mathrm{D}$, see Figure 7. For $\partial \mathrm{D}$ we take again a sphere with infinite radius. Hence,

$$
\begin{aligned}
\hat{\mathbf{G}}\left(\mathbf{x}^{\prime}, \mathbf{x}^{\prime \prime}, \omega\right)= & \hat{\overline{\mathbf{G}}}\left(\mathbf{x}^{\prime}, \mathbf{x}^{\prime \prime}, \omega\right) \\
& -\int_{\mathbb{D}} \hat{\overline{\mathbf{G}}}\left(\mathbf{x}^{\prime}, \mathbf{x}, \omega\right) \Delta \hat{\mathbf{H}}(\mathbf{x}, \omega) \hat{\mathbf{G}}\left(\mathbf{x}, \mathbf{x}^{\prime \prime}, \omega\right) \mathrm{d}^{3} \mathbf{x}
\end{aligned}
$$

with $\Delta \hat{\mathbf{H}}$ defined by equation 55 . The iterative solution of this integral equation is given by

$$
\begin{aligned}
\left\{\hat{\mathbf{G}}\left(\mathbf{x}^{\prime}, \mathbf{x}^{\prime \prime}, \omega\right)\right\}^{(k)}= & \hat{\overline{\mathbf{G}}}\left(\mathbf{x}^{\prime}, \mathbf{x}^{\prime \prime}, \omega\right) \\
& -\int_{\mathbb{D}} \hat{\overline{\mathbf{G}}}\left(\mathbf{x}^{\prime}, \mathbf{x}, \omega\right) \Delta \hat{\mathbf{H}}(\mathbf{x}, \omega) \\
& \times\left\{\hat{\mathbf{G}}\left(\mathbf{x}, \mathbf{x}^{\prime \prime}, \omega\right)\right\}^{(k-1)} \mathrm{d}^{3} \mathbf{x},
\end{aligned}
$$

for $k \geq 1$, with

$$
\left\{\hat{\mathbf{G}}\left(\mathbf{x}^{\prime}, \mathbf{x}^{\prime \prime}, \omega\right)\right\}^{(0)}=\hat{\overline{\mathbf{G}}}\left(\mathbf{x}^{\prime}, \mathbf{x}^{\prime \prime}, \omega\right) .
$$

For $k=1$, equation 78 is the Born approximation, which is frequently used as a representation of primary data in modeling and inversion (Cohen and Bleistein, 1979; Raz, 1981; Bleistein and Cohen, 1982; Tarantola, 1984; Miller et al., 1987; Wu and Toksöz, 1987; Oristaglio, 1989). For $k>1$, equation 78 represents a Neumann series expansion, which can be used for modeling primaries as well as inter-

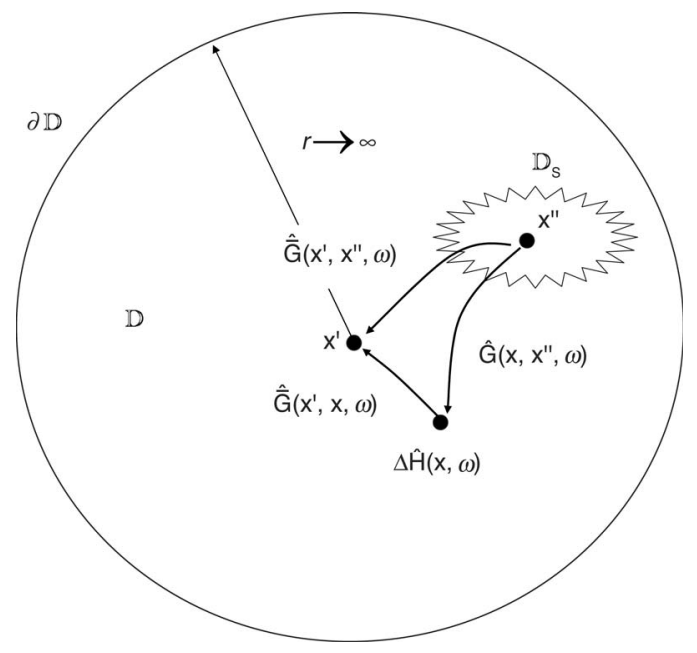

Figure 7. Configuration for volume integral representation.
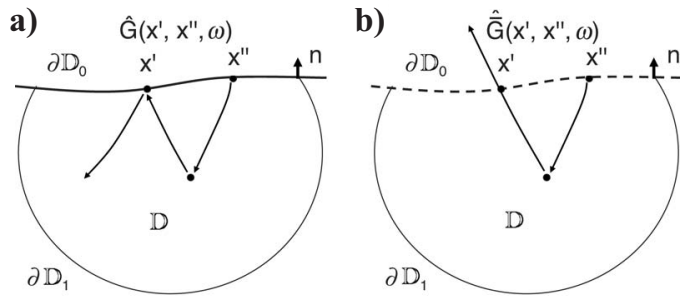

Figure 8. Configuration for multiple elimination. nal multiples. For a discussion on the convergence aspects, see Fokkema and van den Berg (1993). Applications for the prediction of internal multiples in nonlinear inversion are discussed by, e.g., Snieder (1990), Ten Kroode (2002) and Weglein et al. (2003).

\section{Surface-related multiple prediction and elimination (convolution approach)}

Surface-related multiple prediction and elimination was introduced by Berkhout (1985) and Verschuur et al. (1992) and it was based on reciprocity theory by Fokkema and van den Berg (1993) and van Borselen et al. (1996). The latter approach is generalized for the wave phenomena discussed in this paper as follows. Let $\partial \mathrm{D}$ consist of the acquisition surface $\partial \mathrm{D}_{0}$ and a hemisphere $\partial \mathrm{D}_{1}$ with infinite radius in the lower half-space (we assume that, beyond some finite radius, the medium in the lower half-space is homogeneous, isotropic, and nonporous). The Green's matrix $\hat{\mathbf{G}}\left(\mathbf{x}^{\prime}, \mathbf{x}^{\prime \prime}, \omega\right)$ in this configuration (with $\mathbf{x}^{\prime}$ and $\mathbf{x}^{\prime \prime}$ at $\partial \mathbb{D}_{0}$ ) represents the actual data, including the multiples related to $\partial \mathrm{D}_{0}$, see Figure $8 \mathrm{a}$. In the half-space below $\partial \mathrm{D}_{0}$, the reference medium is specified as identical to the actual medium. In the upper half-space, the reference parameters are homogeneous, isotropic, and nonporous, and specified as continuous across $\partial \mathrm{D}_{0}$. Hence, the Green's matrix $\hat{\overline{\mathbf{G}}}\left(\mathbf{x}^{\prime}, \mathbf{x}^{\prime \prime}, \omega\right)$ in the reference medium represents the data without surface-related multiples, see Figure $8 \mathrm{~b}$. The relation between the two Green's matrices follows from equation 54 and is given by

$$
\begin{aligned}
& \hat{\overline{\mathbf{G}}}\left(\mathbf{x}^{\prime}, \mathbf{x}^{\prime \prime}, \omega\right)-\hat{\mathbf{G}}\left(\mathbf{x}^{\prime}, \mathbf{x}^{\prime \prime}, \omega\right) \\
& \quad=\int_{\partial \mathrm{D}_{0}} \hat{\overline{\mathbf{G}}}\left(\mathbf{x}^{\prime}, \mathbf{x}, \omega\right) \mathbf{N}_{\mathbf{x}} \hat{\mathbf{G}}\left(\mathbf{x}, \mathbf{x}^{\prime \prime}, \omega\right) \mathrm{d}^{2} \mathbf{x} .
\end{aligned}
$$

This expression can be used as the basis for modeling as well as elimination of surface-related multiples. For modeling applications, we assume that $\hat{\overline{\mathbf{G}}}\left(\mathbf{x}^{\prime}, \mathbf{x}^{\prime \prime}, \omega\right)$, the response without surface-related multiples, is known. Then $\hat{\mathbf{G}}\left(\mathbf{x}^{\prime}, \mathbf{x}^{\prime \prime}, \omega\right)$, the response with surface-related multiples, can be found by solving equation 80 iteratively, according to

$$
\begin{aligned}
\left\{\hat{\mathbf{G}}\left(\mathbf{x}^{\prime}, \mathbf{x}^{\prime \prime}, \omega\right)\right\}^{(k)}= & \hat{\overline{\mathbf{G}}}\left(\mathbf{x}^{\prime}, \mathbf{x}^{\prime \prime}, \omega\right) \\
& -\int_{\partial \mathrm{D}_{0}} \hat{\overline{\mathbf{G}}}\left(\mathbf{x}^{\prime}, \mathbf{x}, \omega\right) \mathbf{N}_{\mathbf{x}}\left\{\hat{\mathbf{G}}\left(\mathbf{x}, \mathbf{x}^{\prime \prime}, \omega\right)\right\}^{(k-1)} \mathrm{d}^{2} \mathbf{x},
\end{aligned}
$$

for $k \geq 1$. The initial estimate is given by the response without multiples; hence,

$$
\left\{\hat{\mathbf{G}}\left(\mathbf{x}^{\prime}, \mathbf{x}^{\prime \prime}, \omega\right)\right\}^{(0)}=\hat{\overline{\mathbf{G}}}\left(\mathbf{x}^{\prime}, \mathbf{x}^{\prime \prime}, \omega\right) .
$$

When equation 80 is used for multiple elimination, then $\hat{\mathbf{G}}\left(\mathbf{x}^{\prime}, \mathbf{x}^{\prime \prime}, \omega\right)$ is the known response, and equation 80 is solved iteratively, according to 


$$
\begin{aligned}
\left\{\hat{\overline{\mathbf{G}}}\left(\mathbf{x}^{\prime}, \mathbf{x}^{\prime \prime}, \omega\right)\right\}^{(k)}= & \hat{\mathbf{G}}\left(\mathbf{x}^{\prime}, \mathbf{x}^{\prime \prime}, \omega\right) \\
& +\int_{\partial \mathrm{D}_{0}}\left\{\hat{\overline{\mathbf{G}}}\left(\mathbf{x}^{\prime}, \mathbf{x}, \omega\right)\right\}^{(k-1)} \mathbf{N}_{\mathbf{x}} \hat{\mathbf{G}}\left(\mathbf{x}, \mathbf{x}^{\prime \prime}, \omega\right) \mathrm{d}^{2} \mathbf{x},
\end{aligned}
$$

for $k \geq 1$. This time, the initial estimate is given by the response with multiples; hence,

$$
\left\{\hat{\overline{\mathbf{G}}}\left(\mathbf{x}^{\prime}, \mathbf{x}^{\prime \prime}, \omega\right)\right\}^{(0)}=\hat{\mathbf{G}}\left(\mathbf{x}^{\prime}, \mathbf{x}^{\prime \prime}, \omega\right) .
$$

The product under the integral in equation 83 represents a convolution process, producing high-order multiples from primaries and lower-order multiples, which, after addition to the first term, compensate the multiples in $\hat{\mathbf{G}}\left(\mathbf{x}^{\prime}, \mathbf{x}^{\prime \prime}, \omega\right)$.

\section{Surface-related multiple prediction and elimination (correlation approach)}

Schuster (2001) and Berkhout and Verschuur (2003) suggest an alternative to the convolution-based multiple prediction and elimination approach, based on correlations. For the configuration discussed above, assuming in addition that the medium is lossless, we obtain from equation 59

$$
\begin{aligned}
& \mathbf{K}^{\hat{\overline{\mathbf{G}}}^{*}}\left(\mathbf{x}^{\prime}, \mathbf{x}^{\prime \prime}, \omega\right) \mathbf{K}+\hat{\mathbf{G}}\left(\mathbf{x}^{\prime}, \mathbf{x}^{\prime \prime}, \omega\right) \\
& =\int_{\partial \mathrm{D}_{0}} \mathbf{K} \hat{\overline{\mathbf{G}}}^{*}\left(\mathbf{x}^{\prime}, \mathbf{x}, \omega\right) \mathbf{K} \mathbf{N}_{\mathbf{x}} \hat{\mathbf{G}}\left(\mathbf{x}, \mathbf{x}^{\prime \prime}, \omega\right) \mathrm{d}^{2} \mathbf{x} \\
& \quad+\int_{\partial \mathrm{D}_{1}} \mathbf{K} \hat{\overline{\mathbf{G}}}^{*}\left(\mathbf{x}^{\prime}, \mathbf{x}, \omega\right) \mathbf{K N}_{\mathbf{x}} \hat{\mathbf{G}}\left(\mathbf{x}, \mathbf{x}^{\prime \prime}, \omega\right) \mathrm{d}^{2} \mathbf{x}
\end{aligned}
$$

The products under the integrals in equation 85 represent a correlation process, producing primaries and low-order multiples from higher-order multiples. Unlike in the convolution representation, the integral along $\partial \mathbb{D}_{1}$ in equation 85 does not vanish when the medium in the lower half-space is homogeneous, isotropic, and nonporous beyond some finite radius. On the other hand, it vanishes due to scattering loss when the medium in the lower half-space is sufficiently inhomogeneous (Wapenaar, 2006).

\section{Interferometry (correlation approach)}

Seismic interferometry deals with the generation of new seismic responses by cross-correlating wavefield measurements at different receiver positions (Claerbout, 1968; Weaver and Lobkis, 2001; Schuster, 2001; Wapenaar et al., 2002; Campillo and Paul, 2003; Derode et al., 2003; Schuster et al., 2004; Sabra et al., 2005; Draganov et al., 2007). The measurements take place in the actual medium, so, the basic expression for interferometry is obtained by taking the reference state equal to the actual state in the representation of the correlation-type, equation 59 . Using the symmetry properties of $\hat{\mathbf{G}}$, $\hat{\mathbf{A}}, \hat{\mathbf{B}}, \hat{\mathbf{Z}}^{\prime}$, and $\mathbf{N}_{\mathbf{x}}$, this yields

$$
\begin{aligned}
\chi_{\mathrm{D}}\left(\mathbf{x}^{\prime \prime}\right) \hat{\mathbf{G}}\left(\mathbf{x}^{\prime}, \mathbf{x}^{\prime \prime}, \omega\right)+\chi_{\mathrm{D}}\left(\mathbf{x}^{\prime}\right) \hat{\mathbf{G}}^{\dagger}\left(\mathbf{x}^{\prime \prime}, \mathbf{x}^{\prime}, \omega\right) \\
=-\oint_{\partial \mathrm{D}} \hat{\mathbf{G}}\left(\mathbf{x}^{\prime}, \mathbf{x}, \omega\right) \mathbf{N}_{\mathbf{x}} \hat{\mathbf{G}}^{\dagger}\left(\mathbf{x}^{\prime \prime}, \mathbf{x}, \omega\right) \mathrm{d}^{2} \mathbf{x} \\
\quad+\int_{\mathrm{D}} \hat{\mathbf{G}}\left(\mathbf{x}^{\prime}, \mathbf{x}, \omega\right) \Delta \hat{\mathbf{H}}(\mathbf{x}, \omega) \hat{\mathbf{G}}^{\dagger}\left(\mathbf{x}^{\prime \prime}, \mathbf{x}, \omega\right) \mathrm{d}^{3} \mathbf{x} \\
\quad+\int_{\partial \mathrm{D}_{\mathrm{int}}} \hat{\mathbf{G}}\left(\mathbf{x}^{\prime}, \mathbf{x}, \omega\right) \mathbf{K} \Delta \hat{\mathbf{H}}^{\mathrm{b}^{*}}(\mathbf{x}, \omega) \mathbf{K} \hat{\mathbf{G}}^{\dagger}\left(\mathbf{x}^{\prime \prime}, \mathbf{x}, \omega\right) \mathrm{d}^{2} \mathbf{x}
\end{aligned}
$$

with

$$
\begin{gathered}
\Delta \hat{\mathbf{H}}=-2 \omega \Im(\hat{\mathbf{A}})+\hat{\mathbf{B}}+\hat{\mathbf{B}}^{\dagger}, \\
\Delta \hat{\mathbf{H}}^{\mathrm{b}}=\mathbf{M}^{T}\left\{\mathbf{J}-\hat{\mathbf{Z}}^{\dagger} \mathbf{J} \hat{\mathbf{Z}}\right\} \mathbf{M} .
\end{gathered}
$$

Equation 86 is a general representation of the Green's matrix between $\mathbf{x}^{\prime \prime}$ and $\mathbf{x}^{\prime}$ in terms of cross-correlations of observed fields at $\mathbf{x}^{\prime \prime}$ and $\mathbf{x}^{\prime}$ because of sources at $\mathbf{x}$ on the boundary $\partial \mathrm{D}$, on the internal imperfect interfaces $\partial \mathrm{D}_{\text {int }}$, as well as in the domain $\mathbb{D}$. The inverse Fourier transform of the left-hand side is $\chi_{\mathbb{D}}\left(\mathbf{x}^{\prime \prime}\right) \mathbf{G}\left(\mathbf{x}^{\prime}, \mathbf{x}^{\prime \prime}, t\right)$ $+\chi_{\mathrm{D}}\left(\mathbf{x}^{\prime}\right) \mathbf{G}^{T}\left(\mathbf{x}^{\prime \prime}, \mathbf{x}^{\prime},-t\right)$, from which $\mathbf{G}\left(\mathbf{x}^{\prime}, \mathbf{x}^{\prime \prime}, t\right)$ is obtained by taking the causal part (assuming $\mathbf{x}^{\prime \prime}$ is located in D). When the medium and interfaces are lossless, it suffices to have sources on $\partial \mathrm{D}$ only, see Figure 9 . Note that $\partial \mathrm{D}$ is not necessarily a closed surface: When the medium is sufficiently inhomogeneous $\partial \mathrm{D}$ can be an open surface (Wapenaar, 2006). On the other hand, when the medium is dissipative throughout $\mathrm{D}$ and the radius of $\partial \mathrm{D}$ is sufficiently large, the boundary integral vanishes and sources are required throughout $D$ (Snieder, 2006; Snieder et al., 2007).

The application of equation 86 in its current form requires independent measurements of the impulse responses of different types of sources at all $\mathbf{x}$ involved in the integrals. The right-hand side can be modified into a direct cross-correlation (i.e., without the integrals) of diffuse field observations at $\mathbf{x}^{\prime \prime}$ and $\mathbf{x}^{\prime}$, the diffusivity being caused by a distribution of uncorrelated noise sources, either on $\partial \mathbb{D}$ (for lossless media) or in D (for dissipative media) (Wapenaar et al., 2006).

Equation 86 has also important applications in efficient modeling and inversion (van Manen et al., 2005, 2006). As mentioned above, for the lossless situation, only the boundary integral over $\partial \mathbb{D}$ needs to be evaluated. Hence, by modeling the responses of a distribution of

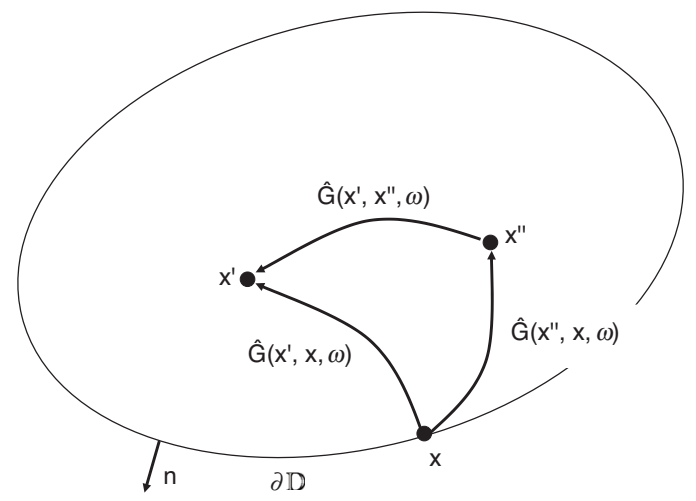

Figure 9. Configuration for interferometry (correlation approach). 


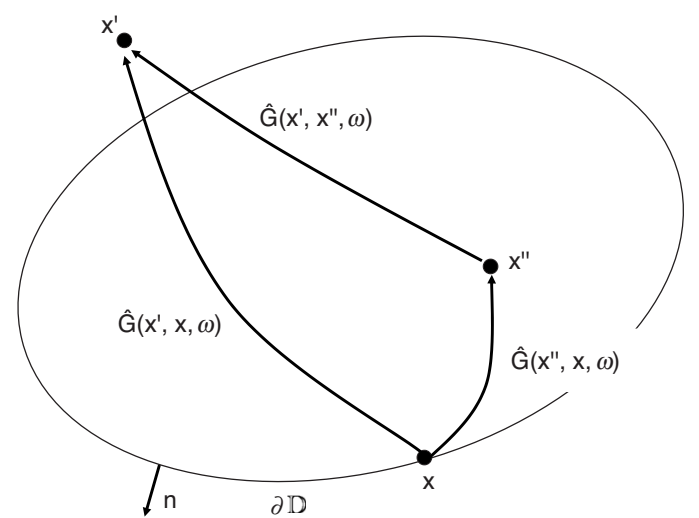

Figure 10. Configuration for interferometry (convolution approach).

sources on the $2 \mathrm{D}$ boundary, equation 86 allows us to determine the responses of all possible sources in the $3 \mathrm{D}$ volume enclosed by the boundary. This is very useful, for example, in nonlinear inversion, where the Green's functions between all possible pairs of points in a volume are needed (see e.g., Weglein et al., 2003).

\section{Interferometry (convolution approach)}

When the dissipation of the medium is significant, interferometry according to the correlation approach requires a distribution of sources throughout the medium. As an alternative, Slob and Wapenaar (2007) and Slob et al. (2007) propose a convolution approach to interferometry. Taking the reference state equal to the real state in the convolution-type representation of equation 54 and using the symmetry property of $\hat{\mathbf{G}}$ gives

$$
\begin{aligned}
& \left\{\chi_{\mathbb{D}}\left(\mathbf{x}^{\prime \prime}\right)-\chi_{\mathrm{D}}\left(\mathbf{x}^{\prime}\right)\right\} \hat{\mathbf{G}}\left(\mathbf{x}^{\prime}, \mathbf{x}^{\prime \prime}, \omega\right) \\
& \quad=\oint_{\partial \mathrm{D}} \hat{\mathbf{G}}\left(\mathbf{x}^{\prime}, \mathbf{x}, \omega\right) \mathbf{N}_{\mathbf{x}} \mathbf{K} \hat{\mathbf{G}}^{T}\left(\mathbf{x}^{\prime \prime}, \mathbf{x}, \omega\right) \mathbf{K} \mathrm{d}^{2} \mathbf{x} .
\end{aligned}
$$

This is a representation of the Green's matrix between $\mathbf{x}^{\prime \prime}$ and $\mathbf{x}^{\prime}$ in terms of cross-convolutions of observed fields at $\mathbf{x}^{\prime \prime}$ and $\mathbf{x}^{\prime}$ due to sources at $\mathbf{x}$ on the boundary $\partial \mathrm{D}$ only. Note that one of the observation points should be inside this boundary and the other outside, see Figure 10 (otherwise, the left-hand side of equation 89 vanishes). There are no restrictions with respect to the losses in the medium. The application of equation 89 requires independent measurements of the impulse responses of different types of sources at all $\mathbf{x} \in \partial \mathrm{D}$; a modification for uncorrelated noise sources is not possible for the convolution approach.

\section{CONCLUSIONS}

Starting with a unified matrix-vector-form wave equation and boundary conditions for acoustic, electromagnetic, elastodynamic, poroelastic, and electroseismic waves, we derived general convolution- and correlation-type wavefield representations. We discussed applications including forward and inverse wavefield extrapolation, boundary integral representations for perfect and imperfect interfaces, volume integral representations (the Born approximation and the Neumann series expansion), multiple elimination, and seismic interferometry, the latter two both in terms of convolutions and correlations. Each of these applications is a generalization of the well-established acoustic representations for any of the wave phenomena governed by the unified wave equation.

\section{ACKNOWLEDGMENTS}

This work is supported by the Netherlands Research Centre for Integrated Solid Earth Science. I thank the editors and reviewers (Roald van Borselen and Enru Liu) for their constructive comments.

\section{APPENDIX A}

\section{THE DIVERGENCE THEOREM OF GAUSS IN MATRIX-VECTOR FORM}

For a scalar field $a(\mathbf{x})$, the divergence theorem of Gauss reads

$$
\int_{\mathrm{D}} \partial_{i} a(\mathbf{x}) \mathrm{d}^{3} \mathbf{x}=\oint_{\partial \mathrm{D}} a(\mathbf{x}) n_{i} \mathrm{~d}^{2} \mathbf{x} .
$$

Here, we modify this theorem for the differential operator matrix $\mathbf{D}_{\mathbf{x}}$ appearing in equations 1 and 9. Let $D_{I J}$ denote the operator in row $I$ and column $J$ of matrix $\mathbf{D}_{\mathbf{x}}$. The symmetry of $\mathbf{D}_{\mathbf{x}}$ (equation 12 ) implies $D_{I J}=D_{J I}$. We define a matrix $\mathbf{N}_{\mathbf{x}}$, which contains the components of the normal vector $\mathbf{n}$, organized in the same way as matrix $\mathbf{D}_{\mathbf{x}}$. Hence, $N_{I J}=N_{J I}$, where $N_{I J}$ denotes the element in row $I$ and column $J$ of matrix $\mathbf{N}_{\mathbf{x}}$. If we replace the scalar field $a(\mathbf{x})$ by $a_{I}(\mathbf{x}) b_{J}(\mathbf{x})$, we may generalize equation A-1 to

$$
\int_{\mathbb{D}} D_{I J}\left\{a_{I}(\mathbf{x}) b_{J}(\mathbf{x})\right\} \mathrm{d}^{3} \mathbf{x}=\oint_{\partial \mathrm{D}} a_{I}(\mathbf{x}) b_{J}(\mathbf{x}) N_{I J} \mathrm{~d}^{2} \mathbf{x},
$$

where the summation convention applies to repeated capital Latin subscripts, which may run from 1 to $4,6,12,16,18$, or 22 , depending on the choice of operator $\mathbf{D}_{\mathbf{x}}$. Applying the product rule for differentiation and using the symmetry property $D_{I J}=D_{J I}$, we obtain for the integrand in the left-hand side of equation A-2,

$$
\begin{aligned}
D_{I J}\left(a_{I} b_{J}\right) & =a_{I} D_{I J} b_{J}+\left(D_{J I} a_{I}\right) b_{J}, \\
& =\mathbf{a}^{T} \mathbf{D}_{\mathbf{x}} \mathbf{b}+\left(\mathbf{D}_{\mathbf{x}} \mathbf{a}\right)^{T} \mathbf{b},
\end{aligned}
$$

where $\mathbf{a}$ and $\mathbf{b}$ are vector functions, containing the scalar functions $a_{I}(\mathbf{x})$ and $b_{J}(\mathbf{x})$, respectively. Rewriting the integrand in the righthand side of equation A-2 in a similar way, we obtain the divergence theorem of Gauss in matrix-vector form

$$
\int_{\mathbb{D}}\left\{\mathbf{a}^{T} \mathbf{D}_{\mathbf{x}} \mathbf{b}+\left(\mathbf{D}_{\mathbf{x}} \mathbf{a}\right)^{T} \mathbf{b}\right\} \mathrm{d}^{3} \mathbf{x}=\oint_{\partial \mathrm{D}} \mathbf{a}^{T} \mathbf{N}_{\mathbf{x}} \mathbf{b} \mathrm{d}^{2} \mathbf{x} .
$$

Finally, we consider a variant of this equation. We replace $\mathbf{a}$ by Ka, where $\mathbf{K}$ is the real-valued diagonal matrix introduced in equations $10-12$, obeying the property $\mathbf{K}=\mathbf{K}^{-1}$. Using equation 12 , we thus obtain

$$
\int_{\mathrm{D}}\left\{\mathbf{a}^{T} \mathbf{K} \mathbf{D}_{\mathbf{x}} \mathbf{b}-\left(\mathbf{D}_{\mathbf{x}} \mathbf{a}\right)^{T} \mathbf{K} \mathbf{b}\right\} \mathrm{d}^{3} \mathbf{x}=\oint_{\partial \mathrm{D}} \mathbf{a}^{T} \mathbf{K} \mathbf{N}_{\mathbf{x}} \mathbf{b} \mathrm{d}^{2} \mathbf{x} .
$$

\section{REFERENCES}

Aki, K., and P. G. Richards, 1980, Quantitative seismology, vol. I: W. H. Freeman \& Company.

Auld, B. A., 1973, Acoustic fields and waves in solids: Wiley-Interscience. Berkhout, A. J., 1985, Seismic migration: Imaging of acoustic energy by wave field extrapolation: Elsevier Science Publ. Co., Inc.

Berkhout, A. J., and D. J. Verschuur, 2003, Transformation of multiples into primary reflections: 73rd Annual International Meeting, SEG, Expanded Abstracts, 1925-1928. 
Biot, M. A., 1956, Theory of propagation of elastic waves in a fluid-saturated porous solid: I - Low frequency range: Journal of the Acoustical Society of America, 28, 168-178.

Bleistein, N., 1984, Mathematical methods for wave phenomena: Academic Press Inc.

, 1987 , On the imaging of reflectors in the earth: Geophysics, 52 , 931-942.

Bleistein, N., and J. K. Cohen, 1982, Velocity inversion - Present status, new directions: Geophysics, 47, 1497-1511.

Bojarski, N. N., 1983, Generalized reaction principles and reciprocity theorems for the wave equations, and the relationship between the time-advanced and time-retarded fields: Journal of the Acoustical Society of America, 74, 281-285.

Born, M., and E. Wolf, 1965, Principles of optics: Pergamon Press, Inc.

Campillo, M., and A. Paul, 2003, Long-range correlations in the diffuse seismic coda: Science, 299, 547-549.

Claerbout, J. F., 1968, Synthesis of a layered medium from its acoustic transmission response: Geophysics, 33, 264-269.

Cohen, J. K., and N. Bleistein, 1979, Velocity inversion procedure for acoustic waves: Geophysics, 44, 1077-1087.

de Hoop, A. T., 1958, Representation theorems for the displacement in an elastic solid and their applications to elastodynamic diffraction theory: Ph.D. thesis, Delft University of Technology.

- 1966, An elastodynamic reciprocity theorem for linear, viscoelastic media: Applied Scientific Research, 16, 39-45.

- 1995, Handbook of radiation and scattering of waves: Academic Press, Inc.

de Hoop, M. V., and A. T. de Hoop, 2000, Wave-field reciprocity and optimization in remote sensing: Proceedings of the Royal Society of London, Series A, 456, 641-682.

Deresiewicz, H., and R. Skalak, 1963, On uniqueness in dynamic poroelasticity: Bulletin of the Seismological Society of America, 53, 783-788.

Derode, A., E. Larose, M. Campillo, and M. Fink, 2003, How to estimate the Green's function of a heterogeneous medium between two passive sensors? Application to acoustic waves: Applied Physics Letters, 83, 3054-3056.

Draganov, D., K. Wapenaar, W. Mulder, J. Singer, and A. Verdel, 2007, Retrieval of reflections from seismic background-noise measurements: Geophysical Research Letters, 34, L04305-1-L04305-4.

Druzhinin, A., H. Pedersen, M. Campillo, and W. Kim, 1998, Elastic Kirchhoff-Helmholtz synthetic seismograms: Pure and Applied Geophysics, 151, 17-45.

Fokkema, J. T., and P. M. van den Berg, 1993, Seismic applications of acoustic reciprocity: Elsevier Science Publ. Co., Inc.

Frazer, L. N., and M. K. Sen, 1985, Kirchhoff-Helmholtz reflection seismograms in a laterally inhomogeneous multi-layered elastic medium, I: Theory: Geophysical Journal of the Royal Astronomical Society, 80, 121-147.

Gangi, A. F., 1970, A derivation of the seismic representation theorem using seismic reciprocity: Journal of Geophysical Research, 75, 2088-2095.

, 2000, Constitutive equations and reciprocity: Geophysical Journal International, 143, 311-318

Gurevich, B., and M. Schoenberg, 1999, Interface conditions for Biot's equations of poroelasticity: Journal of the Acoustical Society of America, $\mathbf{1 0 5}$ 2585-2589.

Hill, N. R., and P. C. Wuenschel, 1985, Numerical modeling of refraction arrivals in complex areas: Geophysics, 50, 90-98.

Kaufman, A. A., and G. V. Keller, 1983, Frequency and transient soundings: Methods in Geochemistry and Geophysics no. 16, Elsevier Science Publ. Co., Inc.

Liu, E., J. A. Hudson, S. Crampin, W. D. Rizer, and J. H. Queen, 1995, Seismic properties of a general fracture, in $\mathrm{H}$. P. Rossmanith, ed., Mechanics of jointed and faulted rock: A. A. Balkema Publishers, 673-678.

Liu, E., J. A. Hudson, and T. Pointer, 2000, Equivalent medium representations of fractured rock: Journal of Geophysical Research-Solid Earth, 105, 2981-3000.

Miller, D., M. Oristaglio, and G. Beylkin, 1987, Anew slant on seismic imaging: Migration and integral geometry: Geophysics, 52, 943-964

Mittet, R., R. Sollie, and K. Hokstad, 1995, Pre-stack depth migration with compensation for absorption and dispersion: Geophysics, 60, 1485-1494.

Morse, P. M., and H. Feshbach, 1953, Methods of theoretical physics, vol. I: McGraw-Hill Book Co. Inc.

Nakagawa, S., K. T. Nihei, and L. R. Myer, 2000, Shear-induced conversion of seismic waves across single fractures: International Journal of Rock Mechanics and Mining Sciences, 37, 203-218.

Oristaglio, M. L., 1989, An inverse scattering formula that uses all the data: Inverse Problems, 5, 1097-1105.

Pao, Y. H., and V. Varatharajulu, 1976, Huygens' principle, radiation conditions, and integral formulations for the scattering of elastic waves: Journal of the Acoustical Society of America, 59, 1361-1371.

Porter, R. P., 1970, Diffraction-limited, scalar image formation with holograms of arbitrary shape: Journal of the Optical Society of America, 60,
1051-1059

Pride, S., 1994, Governing equations for the coupled electromagnetics and acoustics of porous media: Physical Review B, 50, 15678-15696.

Pyrak-Nolte, L. J., L. R. Myer, and N. G. W. Cook, 1990, Transmission of seismic waves across single natural fractures: Journal of Geophysical Research, 95, 8617-8638.

Raz, S., 1981, Three-dimensional velocity profile inversion from finite-offset scattering data: Geophysics, 46, 837-842.

Sabra, K. G., P. Gerstoft, P. Roux, W. A. Kuperman, and M. C. Fehler, 2005, Surface wave tomography from microseisms in Southern California: Geophysical Research Letters, 32, L14311-1-L14311-4.

Schneider, W. A., 1978, Integral formulation for migration in two and three dimensions: Geophysics, 43, 49-76.

Schoenberg, M., 1980, Elastic wave behaviour across linear slip interfaces: Journal of the Acoustical Society of America, 68, 1516-1521.

Schuster, G. T., 2001, Theory of daylight/interferometric imaging: Tutorial: 63rd Annual Conference and Exhibition, EAGE, Extended Abstracts A32-1-A32-4.

Schuster, G. T., J. Yu, J. Sheng, and J. Rickett, 2004, Interferometric/daylight seismic imaging: Geophysical Journal International, 157, 838-852.

Slob, E., and K. Wapenaar, 2007, Electromagnetic Green's functions retrieval by cross-correlation and cross-convolution in media with losses: Geophysical Research Letters, 34, L05307-1-L05307-5.

Slob, E., D. Draganov, and K. Wapenaar, 2007, Interferometric electromagnetic Green's functions representations using propagation invariants: Geophysical Journal International, 169, 60-80.

Snieder, R., 1990, The role of the Born approximation in nonlinear inversion: Inverse Problems, 6, 247-266.

, 2006, Retrieving the Green's function of the diffusion equation from the response to a random forcing: Physical Review E, 74, 046620-1046620-4.

Snieder, R., K. Wapenaar, and U. Wegler, 2007, Unified Green's function retrieval by cross-correlation: Connection with energy principles: Physical Review E, 75, 036103-1-036103-14.

Tarantola, A., 1984, Linearized inversion of seismic reflection data: Geophysical Prospecting, 32, 998-1015.

Ten Kroode, F., 2002, Prediction of internal multiples: Wave Motion, 35, 315-338.

Tygel, M., J. Schleicher, L. T. Santos, and P. Hubral, 2000, An asymptotic inverse to the Kirchhoff-Helmholtz integral: Inverse Problems, 16, $425-445$.

van Borselen, R. G., J. T. Fokkema, and P. M. van den Berg, 1996, Surface-related multiple elimination: Geophysics, 61, 202-210.

van Manen, D.-J., A. Curtis, and J. O. A. Robertsson, 2006, Interferometric modeling of wave propagation in inhomogeneous elastic media using time reversal and reciprocity: Geophysics, 71, SI41-SI60.

van Manen, D.-J., J. O. A. Robertsson, and A. Curtis, 2005, Modeling of wave propagation in inhomogeneous media: Physical Review Letters, 94, 164301-1-164301-4.

Verschuur, D. J., A. J. Berkhout, and C. P. A. Wapenaar, 1992, Adaptive surface-related multiple elimination: Geophysics, 57, 1166-1177.

Wapenaar, K., 2006, Green's function retrieval by cross-correlation in case of one-sided illumination: Geophysical Research Letters, 33, L19304-1L19304-6.

Wapenaar, C. P. A., and A. J. Berkhout, 1989, Elastic wave field extrapolation: Elsevier Science Publ. Co., Inc.

Wapenaar, K., D. Draganov, J. Thorbecke, and J. Fokkema, 2002, Theory of acoustic daylight imaging revisited: 72nd Annual International Meeting, SEG, Expanded Abstracts, 2269-2272.

Wapenaar, K., and J. Fokkema, 2004, Reciprocity theorems for diffusion, flow and waves: Journal of Applied Mechanics, 71, 145-150.

Wapenaar, K., E. Slob, and J. Fokkema, 2004, Reciprocity and power balance for piecewise continuous media with imperfect interfaces: Journal of Geophysical Research -Solid Earth, 109, B10301-1-B10301-9.

Wapenaar, K., E. Slob, and R. Snieder, 2006, Unified Green's function retrieval by cross correlation: Physical Review Letters, 97, 234301-1234301-4.

Weaver, R. L., and O. I. Lobkis, 2001, Ultrasonics without a source: Thermal fluctuation correlations at MHz frequencies: Physical Review Letters, 87, 134301-1-134301-4.

Weglein, A. B., F. V. Araújo, P. M. Carvalho, R. H. Stolt, K. H. Matson, R. T. Coates, D. Corrigan, D. J. Foster, S. A. Shaw, and H. Zhang, 2003, Inverse scattering series and seismic exploration: Inverse Problems, 19, R27-R83.

Wenzel, F., K. J. Stenzel, and U. Zimmerman, 1990, Wave propagation in laterally heterogeneous layered media: Geophysical Journal International, 103, 675-684

Wu, R. S., and M. Toksöz, 1987, Diffraction tomography and multisource holography applied to seismic imaging: Geophysics, 52, 11-25.

Zhang, J., and K. Wapenaar, 2002, Wavefield extrapolation and prestack depth migration in anelastic inhomogeneous media: Geophysical Prospecting, 50, 629-643. 